(C) 2017 IEEE. Personal use of this material is permitted. Permission from IEEE must be obtained for all other uses, in any current or future media, including reprinting/republishing this material for advertising or promotional purposes, creating new collective works, for resale or redistribution to servers or lists, or reuse of any copyrighted component of this work in other works. 


\title{
Finding the Right Tree: Topology Inference Despite Spatial Dependencies
}

\author{
Rhys Bowden Darryl Veitch (FIEEE) \\ University of Melbourne University of Technology Sydney
}

\begin{abstract}
Network tomographic techniques have almost exclusively been built on a strong assumption of mutual independence of link processes. We introduce model classes for link loss processes with non-trivial spatial dependencies, for which the tree topology is nonetheless identifiable from leaf measurements using multicast probing. We show that these classes are large in a well defined sense, and we provide an algorithm, SLTD2, capable of returning the correct topology with certainty in the limit of infinite data.
\end{abstract}

\section{Index Terms}

Network tomography, spatial dependence, identifiability, topology inference, loss measurement, multicast-trees.

\section{INTRODUCTION}

$\mathbf{N}$ ETWORK Tomography is concerned with inferring underlying details of networks from incomplete or inaccurate measurements. In network link tomography over trees, a unique sender node sends many test packets, or probes, to $m>1$ receiver nodes. The design of network routing is such that, typically, the sequences of network links traversed by such probes will naturally possess a tree topology, a measurement tree subgraph of the underlying network graph. Each receiver records measurements from the probes that arrive to it, and these are used to infer conditions at links or nodes inside the tree.

Particular attention has been paid to multicast-trees. In multicasting, a packet sent from a source toward $m$ destinations is duplicated only when needed, at branch points, rather than the source sending $m$ copies. In multicast-tree tomography this functionality is abstracted into the powerful property of perfectly shared-history of receiver probes above branch points. Multicast-tree tomography can be seen both as an exploitation of IP protocol functionality for measurement ends [1]-[9], or as a means to infer properties of a multicast-tree to improve the performance of applications using it [10]. It can also be viewed as a simplifying abstraction to explore the limitations of tree-based tomography in general, in particular because unicast approaches exist which can emulate the shared-history property of multicast.

Tree-based tomography has considered inference of link loss processes (e.g. [2], [11])), link delays (e.g. [4], [6])), and tree topology (e.g. [7], [10], [12], [13]). In almost every case full spatial independence, that is the mutual independence of all loss or delay processes across different links, was a core assumption underlying the inference. We refer to any model incorporating this classical assumption as a classical model. Two works where non-classical models are considered are those of Caceras et al. [2] and Ghita et al. [14], both in the context of loss tomography. We discuss these in Section II.

This paper describes loss-based topology inference in multicast-trees, in the context of non-trivial spatial dependence of link loss processes. In order to focus on fundamental issues of identifiability, we work in the ideal context of 'infinite data', meaning we have access to the true $m$ dimensional joint distribution of loss measurements across all leaves/receivers.

Our first contribution lies in the definition of new model classes with non-trivial spatial dependencies. The significance of these classes is that they each possess the non-trivial property of topological determinism, which implies that the topology of each model within the class is identifiable, even if the full model itself is not. We begin by defining a natural generalization of classical models, the Classically Equivalent (CE) class. We then introduce a more explicit and richer class, the Jump Independent (JI) models, which is physically meaningful in terms of real networks, and from it define two related classes, the Agreeable JI models (AJI), and the Agreeable JI Equivalent models (AJIE), which are topologically determinate. We show that each is large in the sense of the dimensionality of its parameter space, and much larger than the classical class, and also that, although their topologies are identifiable, the loss processes on links are not. Figure 6 shows the relationship between these classes.

Our second contribution is in the definition of topology recovery algorithms and proofs of their correctness. We first describe Shared Loss Topology Discovery (SLTD), which is capable of fully identifying the topology of CE models without error. This SLTD algorithm was inspired by, and is closely related to, that proposed in [7], [10] for topology inference under spatial independence. We show how, in the infinite data case, it is based on a property we call 'certain paternity' which is powerful enough to allow SLTD to be applied much more widely.

We then describe another algorithm, SLTD2, designed to recover the topology of models from the AJI class, a far more challenging task. It is also based on shared loss between receiver pairs, but using a different and more general certain paternity property. Using an approach of broad applicability, we prove that SLTD2 also recovers the topology of models in the AJIE class, which includes the CE class as a special case. Hence SLTD2 is a new algorithm that can be used instead of SLTD but with much wider applicability. Importantly, it is based on the joint distributions of pairs of receivers only, yet captures 
everything that is possible even given the full $m$ dimensional joint distribution over all receivers. It is therefore amenable to practical implementation as there is no need to estimate densities of dimension exceeding 2.

The remainder of the paper is structured as follows. In Section II we motivate our problem and describe related work. In Section III we define multicast-trees and processes on them, Section IV classifies these tree processes and discuss identifiability issues, and Section V defines SLTD and establishes its properties. In Section VI we introduce Classical Equivalence, prove its key properties, and show that SLTD can recover the topology of such models. In Section VII we introduce the Jump Independent model class and establish its key properties. Section VIII shows how to prove 'certain paternity' for AJI models, and based on this, defines the SLTD2 algorithm to enable topology recovery for AJI models, then proves that the algorithm also works for the larger AJIE class. In Section IX the relative size of the CE, JI and other classes is discussed, and some examples given for small topologies. We conclude in Section X.

\section{Modelling Motivation And Relationship to PREVious WORK}

\section{A. Spatial Dependency}

A natural question is why it is necessary to consider network spatial dependencies. Network tomography is impossible without a spatial model, yet empirical insight into this question is not readily available, and the data required is difficult to collect. In its absence, the literature has mainly adopted the classical assumption of full spatial independence, which enjoys the two important advantages of tractability and parameter identifiability.

There are two canonical reasons why spatial independence will in fact fail to hold in general. The first is that networks defined in tomographic problems are generally logical, that is they describe connectivity between nodes, but not how it is established. As pointed out by Ghita et al. [14], the hidden underlying physical connectivity can involve shared resources which will induce spatial dependency in the logical links. For example in the Internet, distinct IP links (layer 3 links in the protocol hierarchy) will be dependent if they share, as they often do, underlying 'link layer' links (layer 2), as illustrated in Figure 1. The resulting dependencies can be far from negligible, for example if the physical link $(a, d)$ is congested.

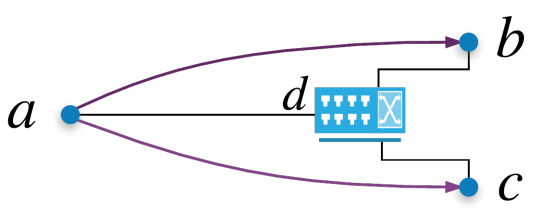

Fig. 1. Spatial dependency in logical networks: the direct logical links $(a, b)$ and $(a, c)$ appear distinct, but can be spatially dependent because of the shared link $(a, d)$ to a switch providing the actual connectivity, even if the losses over $(a, d)$ and the other two links at the lower layer are mutually independent.

The second canonical mechanism is due to resource sharing at the logical level. Dependence is created in switching equipment such as IP routers because both the probe and cross (non-probe) traffic flows share memory and computation resources, and contend for access to output ports. Spatial dependencies in traffic flows across multiple shared links is thereby induced by temporal dependencies in the cross traffic flows modulating resource levels at each link (see [14] for a discussion). For the same reason, spatial dependencies between probes on sibling links are generated when temporally correlated cross traffic flows branch out from the parent link and influence the conditions experienced by the separated probes. These effects were discussed in [2] in the context of TCP flow synchronization, and the resulting dependencies exhibited in simulations.

In the case of multicast probing there is a third mechanism. Network tomography problems are typically posed based on simplified statistical models for tractability, but the measurement tree is more properly modelled as a feedforward queueing network. In this context we showed in [15] (for delay tomography) that even in the most favorable case of independent exponentially distributed service times and independent Poisson arrival processes for all cross traffics, the use of multicasting generated inherent spatial dependencies between sibling links of branch nodes. From this perspective, the models introduced in the present paper, which explicitly allow for such dependency, are necessary for a multicast based tomographic problem which is self-consistent.

The spatial independence assumption is likely to be an increasingly poor approximation in the future. One reason for this is the spread of Software Defined Networking (SDN), which allows adaptive flow control rules to be readily defined and applied on a per-node basis. In such an environment, even if spatial dependency data were obtained, it would be subject to ongoing change. The models we propose are general, allowing arbitrary local spatial dependency at each network node. Topology tomographic methods built on the class will therefore be robust to the particular dependencies that may exist in real networks at a given time, without having to be aware of their detailed nature.

\section{B. Multicast Probing}

Multicast is supported by the Internet's IP protocol suite and is implemented in the current Internet. However, as it is not necessarily activated, it remains difficult today to perform multicast based measurement, particularly across different 
administrative domains. This has motivated a body of work on unicast approaches to emulate the shared-history property of multicast, including [11], [13], [16]-[20]. Multicast based tomography remains valuable for three reasons.

First, multicast support will inprove in the future. It is obligatory in all nodes supporting IPv6 (version 6 of the IP protocol), and now that IPv4 addresses have been exhausted, widespread IPv6 deployment is picking up speed. More flexible networking approaches such as SDN will also make it easier to put in place and activate multicasting.

Second, unicast approaches can be brought under our JI model framework. By replacing a multicast probe by a train of probes (one for each receiver), we can show (based on assumptions consistent with our JI modelling principles) that both the key Fundamental Property of JI Models (Theorem 4) and the 'Shared world-view property of siblings' (Lemma 3) continue to hold. However, this comes at the cost of considerable complexity.

Another alternative is to use the 'Boolean network' approach [14], [21]-[24], that allows the shared-history property of multicasting to be recovered at a higher level, despite the use of underlying unicast probe trains. This is achieved by introducing the concept of 'congested' links and paths based on loss rates exceeding controllable thresholds, over timescales beyond that of individual probe trains. In particular the set of assumptions adopted by Ghita et al. [14] result in a mathematical framework identical to that of multicasting (as noted in [21]).

Finally, multicast based inference provides a bound on what is achievable for tomographic methods more generally.

\section{Related Work}

The seminal multicast-tree tomography paper of Caceres et al. [2] examines loss tomography (inferring loss metrics with fixed known topology) rather than topology tomography. It is nonetheless of interest here because, although primarily based on classical assumptions, it includes elements examining spatial dependency. One section uses network protocol and queueing based simulation. It is reported that this generates spatial dependencies (via the second and third canonical mechanisms above), that cause bias in estimators of marginal link loss probabilities under classical assumptions. In the special case of binary trees with dependent siblings, another theoretical section shows how these estimators could be corrected, if side information characterizing the dependencies were known. This demonstrates that even the simplest examples of dependency prevent identifiability of link loss probabilities.

In Ghita et al. [14] (see also [23]) non-classical dependency is the focus. Their setting is that of loss tomography in general networks, and is based on a critical identifiability assumption involving the selection of sets of links, called correlation sets, that obey a distinguishable path property enabling their independent measurement. They also perform simulation studies with spatial dependencies and report that ignoring them results in significant error. In trees however, the identifiability assumption restricts correlation sets strongly, essentially because trees lack the topological diversity required to easily isolate set of links from each other. The correlation sets allowed in trees do not in general respect the physical network principles described in Section II-A. For example they cannot contain a complete group of sibling links as JI models do, nor can a correlation set contain the father or child of any other link in the set. They are therefore of limited interest in trees. Moreover, their method cannot be used for topology tomography as the correlation sets must be identified prior to inference, when in topology tomography which links exist isn't even known prior to inference taking place.

Beyond the works cited in the introduction, more recent topology tomography research has also assumed spatial independence [25], combined with other sources of information like network coding [26] or traceroute and record route messages [27], [28]. $\mathrm{Ni}$ et al. [28] state that spatial independence is not realistic in the Internet, and address it by introducing a hybrid method where they place greater emphasis on alternative traceroute information. Many methods focus on using additive metrics like shared loss (Section V) that increase down a path, allowing those with the longest shared path to be identified as siblings [25], [28]. In the models that we propose here, shared loss is no longer additive (Section VII) so these approaches no longer work. The work closest to allowing spatial dependence is provided by Eriksson et al. [29], who only require a monotonicity condition related to their DFS (Depth First Search) algorithm.

Finally, we comment on the relation between our models and those studied in graphical models and Bayesian networks, that employ conditional independence relationships reminiscent of those defining JI models. Our problem is analogous to learning a graphical model with unknown structure, and an unknown number of latent variables/nodes, but with additional sibling dependencies. The field of hierarchical latent class models (HLCs) and its generalization, latent-variable tree models (LTMs) [30]-[32] is the most similar to JI models. However, there are some major differences and typical approaches from that area do not work here.

In latent tree models and graphical models in general, child nodes are conditionally independent given the value of the parent node. In JI models, this is explicitly not the case. Further, common means of grouping variables, e.g. mutual information [33] prove ineffective in this context, even when applied to classical models, because they do not exploit the inherent multiplicative structure of loss modelling. Note that the Classically Equivalent models (Section VI) can have their output distribution modelled by an LTM with a direct correspondence between the topology of the LTM and the true topology, simply because the equivalent classical model is an LTM. For JI models outside of CE this is not possible, and any attempt to bundle the spatial dependencies into fewer nodes with non-binary state destroys the powerful multiplicative property which underpins the inference. In the general case of non-binary trees, $\mathrm{CE} \cap \mathrm{JI}$ is negligible in size compared to JI (Section IX), and so LTMs are not relevant for 
JI models in general. Finally, the LTM literature is focused on issues pertaining to finite data inference which do not arise, and are not helpful, in the infinite data case.

\section{Notation: Trees And Loss Processes}

Consider a multicast-tree $T=(V, L)$ where $V$ is the set of nodes and $L$ the set of $n=|L|$ links. Let $0 \in V$ be the root node, and $R \subset V$ be the set of leaf nodes (receivers). Each link is an ordered pair $(k, j) \in V \times V$ representing a logical link (one or more physical links) from node $k$ to node $j$. Let $c(k)=\{j \in V \mid(k, j) \in L\}$, the set of children of node $k$, and let its degree be $|c(k)|$. Each node has at least two children except the root and leaves. For each $k \in V \backslash 0$ let $f(k)$ denote the parent of $k$, the unique node such that $(f(k), k) \in L$. Define $f(0)=0$, and refer to link $(f(k), k)$ as link $k$ (there is no link $0)$. Denote the set of ancestors of $k$ by $a(k)=\bigcup_{n>1} f^{n}(k)$ and descendants by $d(k)=\{j \in V \mid k \in a(j)\}$. If $j \in a(k)$, then path from $j$ to $k, j \rightarrow k$, is the sequence of links from node $j$ to node $k$ (note this does not include link $j$ ).

An infinite sequence of probes $i=1,2 \ldots$ are sent from the root to all receivers. The probing is multicast, so when a probe reaches node $k$ a copy is sent on to each of $k$ 's children. Define the link passage processes

$$
Z_{k}(i)= \begin{cases}1 & \text { if probe } i \text { would successfully traverse link } k \\ \text { were it to reach that link; } & \\ 0 & \text { if probe } i \text { would be lost upon reaching link } k .\end{cases}
$$

Note that it is more convenient to deal with passage, or transmission, than dealing directly with its complement, loss.

Denote the marginal link passage probability on link $k$ as $l_{k}=\operatorname{Pr}\left(Z_{k}=1\right)$. We assume that no link has perfect transmission, so $l_{k}<1$ for all $k$, since perfect links effectively disappear from the topology, they are not identifiable.

The link passage processes induce a path passage process:

$$
X_{k}(i)= \begin{cases}1 & \text { if probe } i \text { reaches node } k \in V \\ 0 & \text { if it is lost prior to reaching node } k .\end{cases}
$$

Thus

$$
X_{k}(i)=\bigcap_{j \in a(k) \cup k}\left\{Z_{j}(i)=1\right\}=\prod_{j \in a(k) \cup k} Z_{j}(i)
$$

It is also convenient to define $Y_{b \rightarrow j}=\prod_{v \in b \rightarrow j} Z_{v}$.

It is convenient to view the link passage processes collectively as a vector-valued stochastic process $\mathbf{Z}(i)=\left[Z_{1}(i), \ldots, Z_{n}(i)\right]$, which we assume to be stationary and ergodic. The spatial dependency structure across the tree is now captured by the form of the marginal $\mathbf{Z}=\left[Z_{1}, \ldots, Z_{n}\right]$ of $\mathbf{Z}(i)$. Since the $Z_{k}$ are $\{0,1\}$-valued variables, the joint density $f_{\mathbf{Z}}$ of $\mathbf{Z}$ consists of the $2^{n}$ probability values

$$
\operatorname{Pr}(\mathbf{Z}=\mathbf{r})=\operatorname{Pr}\left(Z_{1}=r_{1}, Z_{2}=r_{2}, \ldots Z_{n}=r_{n}\right),
$$

one for each link passage pattern $\mathbf{r}=\left[r_{1}, \ldots, r_{n}\right] \in\{0,1\}^{n}$. These values sum to 1 , and so provide only $2^{n}-1$ degrees of freedom. We assume that each probability is strictly between 0 and 1 , a natural generalization of the assumption $0<l_{k}<1$. It is not difficult to show that passage probabilities are sufficient. That is, $f_{\mathbf{Z}}$ can also be specified through the probabilities

$$
\operatorname{Pr}\left(\mathbf{Z}_{I}=\mathbf{1}_{|I|}\right)=\operatorname{Pr}\left(\bigcap_{i \in I}\left\{Z_{i}=1\right\}\right),
$$

where $I \subset\{1, \ldots, n\}$ denotes some non-null subset of indices and $\mathbf{1}_{|I|}$ is the length $|I|$ vector of ones.

The path passage processes are also binary, and can be viewed collectively as a stationary and ergodic vector process $\mathbf{X}(i)$ with marginal $\mathbf{X}$, whose density $f_{\mathbf{X}}$ can be characterized just as for $\mathbf{Z}$ above. We are particularly interested in the path passage processes at the leaves, as these correspond to the observables of a measurement experiment. Thus we define $\mathbf{X}_{R}(i)=\left[X_{k}(i)\right]_{k \in R}$, with marginal $\mathbf{X}_{R}$ having density $f_{\mathbf{X}_{R}}$, or $f_{R}$ for short. As there are $m$ leaves, $f_{R}$ has at most $2^{m}-1$ degrees of freedom. As stated in the introduction, we assume we have access to a sufficiently large number of probes to consider that $f_{R}$ is known to arbitrary accuracy. We do not however assume that we know the entire process. Thus the temporal structure of $\mathbf{X}_{R}(i)$, although in general non-trivial since that of $\mathbf{Z}(i)$ is general, is not exploited. We consider inference based on the vector marginal $f_{R}$ only.

An important special case is when the link loss processes are spatially and temporally independent of each other. Under these assumptions the marginal passage probabilities $\left\{l_{k}\right\}$, together with the underlying topology of the tree, are sufficient to entirely determine the tree loss process $\mathbf{Z}(i)$ and hence $f_{\mathbf{Z}}$. Under these classical assumptions, the dimension of the parameter space for $\mathbf{Z}$ is now $n$ instead of $2^{n}-1$. 


\section{Measurement Equivalence \& Identifiability}

We call a tree topology $T$, together with a joint distribution $f_{\mathbf{Z}}$, a model $M=\left(T, f_{\mathbf{Z}}\right)$. A model $M$ induces a joint distribution $f_{R}(M)$ on the vector observable $\mathbf{X}_{R}$. We denote by $\mathcal{M}$ (resp. $\mathcal{M}_{\mathrm{C}}$ ) the set of all (resp. classical) models.

We say that two models $M_{1}$ and $M_{2}$ with the same number of receivers are measurement equivalent if $f_{R}\left(M_{1}\right)=f_{R}\left(M_{2}\right)$. As we show formally later, this is not possible within classical models: each possible $f_{R}$ corresponds to only one $M_{\mathcal{C}} \in \mathcal{M}_{\mathrm{C}}$, which means that both its topology $T(M)$, and distribution $f_{\mathbf{Z}}(M)$, are identifiable from $f_{R}(M)$ (indeed in prior finite-data work one typically estimates them jointly). As soon as spatial dependency is added however this fails, and typically neither $f_{\mathbf{Z}}$ nor $T$ are identifiable. An explicit example of this is given at the end of this section.

The novel question we ask here is, if we are no longer concerned with identifiability of $f_{\mathbf{Z}}$, then under what circumstances can $T$ nonetheless be identified, and can it be done for spatial dependencies which are both strong and physically meaningful? In fact, identifiability is not a property of an individual model, but of a set (class) of models. An obvious necessary condition such a class must obey is the following.

Definition 1 (Topologically Determinate). A set $\mathcal{M}_{1} \subset \mathcal{M}$ is topologically determinate if models $M_{1}\left(T_{1}, f_{1}\right), M_{2}\left(T_{2}, f_{2}\right) \in$ $\mathcal{M}_{1}$ are measurement equivalent only if $T_{1}=T_{2}$.

Clearly $\mathcal{M}_{\mathrm{C}}$ is topologically determinate, however it is a small and spatially trivial class. In Section VII we define much larger and richer topologically determinate classes.

We conclude by showing how the non-identifiability of $f_{\mathbf{z}}$, combined with topological determinism, can actually be exploited, in a very simple but powerful way, to extend topologically identifiable classes, and to extend/reveal the applicability of existing algorithms.

Let $\mathcal{F}_{m}$ be the set of joint distributions on $m$ binary variables, and $\mathcal{T}_{m}$ the set of tree topologies with $m$ receivers. Then a topology discovery algorithm $A$ is a collection $\left\{A_{m}\right\}_{m \geq 1}$ of functions mapping observed distributions to topologies, where for a vector observable of length $m$ the algorithm applies $A_{m}: \mathcal{F}_{m} \rightarrow \mathcal{T}_{m} \cup \emptyset$. Here an output of $\emptyset$ indicates the algorithm exits with error due to input detected as unacceptable. We write $T(M)$ to mean the tree component of the model $M$.

Proposition 1. Let $\mathcal{M}_{1} \subset \mathcal{M}$ be topologically determinate. Let $A$ be a topology recovery algorithm. If $\forall f \in f_{R}\left(\mathcal{M}_{1}\right)$ $\exists M \in \mathcal{M}_{1}$ such that $f_{R}(M)=f$ and $A(f)=T(M)$, then $\forall M \in \mathcal{M}_{1}, A\left(f_{R}(M)\right)=T(M)$.

In other words, if there is at least one model that $A$ works on within each measurement equivalence class in $\mathcal{M}_{1}$, then $A$ establishes the correct topology for all models in $\mathcal{M}_{1}$. The proof is straightforward.

Proof. Take any $M \in \mathcal{M}_{1}$. Since $f_{R}(M) \in f_{R}\left(\mathcal{M}_{1}\right)$, by the assumption $\exists M^{\prime} \in \mathcal{M}_{1}$ such that $f_{R}\left(M^{\prime}\right)=f_{R}(M)$ and $A\left(f_{R}\left(M^{\prime}\right)\right)=T\left(M^{\prime}\right)$. Since $\mathcal{M}_{1}$ is topologically determinate, $f_{R}\left(M^{\prime}\right)=f_{R}(M)$ implies $T\left(M^{\prime}\right)=T(M)$, so $A\left(f_{R}(M)\right)=$ $A\left(f_{R}\left(M^{\prime}\right)\right)=T\left(M^{\prime}\right)=T(M)$ as required.

Example 1. Consider a model $M_{1}$ with the topology on the left in Figure 2, with $f_{\mathbf{Z}}$ obeying classical assumptions with link passage probability $l_{k}=0.9$ for each link $k$. Let model $M_{2}$ have the topology on the right in the figure. We first specify those passage probabilities of $f_{\mathbf{Z}}\left(M_{2}\right)$ which determine the $2^{3}-1=7$ passage probabilities of the observables, and set them to be the same as for $M_{1}$ :

$$
\begin{aligned}
& \operatorname{Pr}\left(X_{1}=1\right)=0.9^{3}=\operatorname{Pr}\left(\left[Z_{1}, Z_{5}\right]=\mathbf{1}_{2}\right) \\
& \operatorname{Pr}\left(X_{2}=1\right)=0.9^{3}=\operatorname{Pr}\left(\left[Z_{2}, Z_{4}, Z_{5}\right]=\mathbf{1}_{3}\right) \\
& \operatorname{Pr}\left(X_{3}=1\right)=0.9^{2}=\operatorname{Pr}\left(\left[Z_{3}, Z_{4}, Z_{5}\right]=\mathbf{1}_{3}\right) \\
& \operatorname{Pr}\left(\left[X_{1}, X_{2}\right]=\mathbf{1}_{2}\right)=0.9^{4}=\operatorname{Pr}\left(\left[Z_{1}, Z_{2}, Z_{4}, Z_{5}\right]=\mathbf{1}_{4}\right) \\
& \operatorname{Pr}\left(\left[X_{1}, X_{3}\right]=\mathbf{1}_{2}\right)=0.9^{4}=\operatorname{Pr}\left(\left[Z_{1}, Z_{3}, Z_{4}, Z_{5}\right]=\mathbf{1}_{4}\right) \\
& \operatorname{Pr}\left(\left[X_{2}, X_{3}\right]=\mathbf{1}_{2}\right)=0.9^{4}=\operatorname{Pr}\left(\left[Z_{2}, Z_{3}, Z_{4}, Z_{5}\right]=\mathbf{1}_{4}\right) \\
& \operatorname{Pr}\left(\mathbf{X}_{R}=\mathbf{1}_{3}\right)=0.9^{5}=\operatorname{Pr}\left(\mathbf{Z}=\mathbf{1}_{5}\right) .
\end{aligned}
$$

Next, set the elementary probabilities $\operatorname{Pr}\left(\mathbf{Z}_{I}=\mathbf{z}_{I}\right)$ freely subject to the above. For example one could choose

$$
\operatorname{Pr}(\mathbf{Z}=\mathbf{z})= \begin{cases}0 & {\left[z_{1}, z_{4}, z_{5}\right]=[1,0,1]} \\ 0.9^{3} 0.1^{2}+0.9^{2} 0.1 & \mathbf{z}=[0,0,1,1,1] \\ 0.9^{\sum_{i} z_{i}} 0.1^{5-\sum_{i} z_{i}} & \text { otherwise. }\end{cases}
$$

where the four elementary probabilities corresponding to $\operatorname{Pr}\left(\left[Z_{1}, Z_{2}, Z_{3}\right]=[1,1,0]\right)$ and the one elementary probability $\operatorname{Pr}(\mathbf{Z})=[1,0,1,0,1]$, take non-classical values (i.e. values inconsistent with any classical model). Hence $M_{2} \notin \mathcal{M}_{\mathrm{C}}$, but is measurement equivalent to $M_{1} \in \mathcal{M}_{\mathrm{C}}$, so any algorithm that is successful on $M_{1}$ will fail on $M_{2}$. 

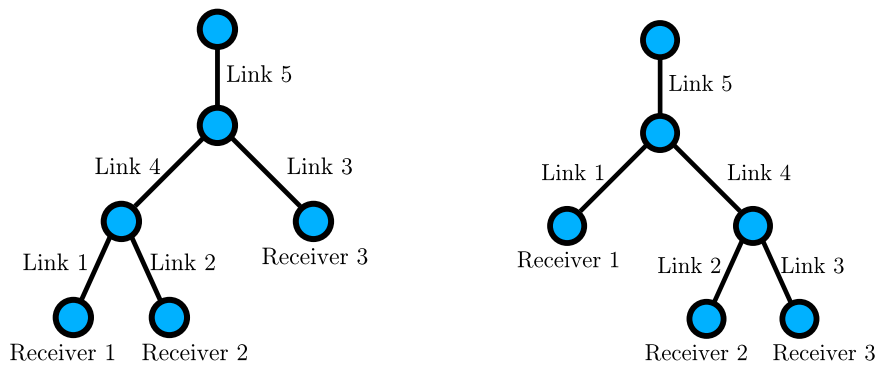

Fig. 2. Tree topologies for Example 1. Left: for $M_{1}$, right $M_{2}$.

\section{Shared Loss Topology Discovery}

We present SLTD, an algorithm designed to recover the topology of classical models. It is closely related to the infinite data algorithm, DLT, of Duffield et al. [7], inspired by the finite data algorithm described in [10]. We revisit it here because, although designed for classical models, it in fact has wider applicability. This section serves to define the terminology and approach that we will subsequently generalize.

The paths $0 \rightarrow j$ and $0 \rightarrow k$ down to nodes $j$ and $k$ intersect along a shared path, $0 \rightarrow b$, terminating at the branching node $b=b_{j, k}$. Under classical assumptions the Shared Transmission

$$
S_{j, k}=\frac{\operatorname{Pr}\left(X_{j}=1\right) \operatorname{Pr}\left(X_{k}=1\right)}{\operatorname{Pr}\left(X_{j}=1 \wedge X_{k}=1\right)}
$$

is of central interest because $S_{j, k}=\operatorname{Pr}\left(X_{b}=1\right)$.

A natural first step to topology inference is to attempt to group receivers into siblings (a bottom-up approach). The following property enables this to be done without error. Recall $Y_{b \rightarrow j}=\prod_{v \in b \rightarrow j} Z_{v}$.

Lemma 1 (Certain Paternity). Assume a classical model, and let $S^{*}=\min _{\{i, l\} \subset R} S_{i, l}$. If $j, k \in R$ obey $S_{j, k}=S^{*}$, then $j$ and $k$ are siblings.

Proof. Assume that $S_{j, k}=S^{*}$ but that $j$ and $k$ are not siblings. Then the branch node $b_{j k}$ lies above at least one of $f(j)$ and $f(k)$. Without loss of generality assume the former. Then a true sibling $i$ of $j$ has shared passage probability $S_{j, i}=S_{j, k} \operatorname{Pr}\left(Y_{b_{j k} \rightarrow f(i)}=1\right)<S_{j, k}=S^{*}$ since no links are lossless, a contradiction.

We now define SLTD. The algorithm is bottom up, starting with a forest of trivial trees, each a single receiver node. At each step, a subset of trees are grouped together (based on certain paternity of their root nodes) into one larger tree by attaching their root nodes as children of a newly created 'virtual' root node. A virtual node $j$ is assigned an inferred path passage $\widetilde{X}_{j}(i)$, with shared transmission between pairs of virtual nodes given by

$$
\widetilde{S}_{j, k}=\frac{\operatorname{Pr}\left(\widetilde{X}_{j}=1\right) \operatorname{Pr}\left(\widetilde{X}_{k}=1\right)}{\operatorname{Pr}\left(\widetilde{X}_{j}=1 \wedge \widetilde{X}_{k}=1\right)} .
$$

Theorem 1 (Correctness of SLTD on classical models).

Let $M=(T, f)$ be a model in $\mathcal{M}_{\mathrm{C}}$. Then SLTD returns $T$.

Proof. Certain paternity holds at the first iteration as all nodes are receivers. Since correct pairwise paternity identification implies correct identification over the sibling set $J$ (true siblings respect transitivity), the algorithm is correct at the first iteration and the error condition is not met. This is also true for subsequent iterations with certain paternity based on $\widetilde{S}_{j, k}$, since for classical models (see proof of Lemma 2) $\widetilde{S}_{j, k}=S_{j, k}$. The algorithm is therefore correct at each iteration and so returns $T$.

It follows from $S_{j, k}=\operatorname{Pr}\left(X_{b_{j, k}}=1\right)$ that path passage probabilities to all nodes in the tree are identifiable. With the topology known via SLTD, the link parameters are then simply given by $l_{k}=\operatorname{Pr}\left(Z_{k}=1\right)=\operatorname{Pr}\left(X_{k}=1\right) / \operatorname{Pr}\left(X_{f(k)}=1\right)$.

Theorem 2 (Classical models are fully identified by SLTD). Any model $M(T, f) \in \mathcal{M}_{\mathrm{C}}$ is identifiable based on knowledge of $f_{R}(M)$. In particular, $\mathcal{M}_{\mathrm{C}}$ is topologically determinate. Moreover SLTD is guaranteed to recover both $T$ and $f$.

In fact SLTD is not restricted to classical models (see next section). It will return the correct topology for many non-classical models, but it may also fail. There are two failure modes. First, SLTD may return a non-null topology which is incorrect (fail silently). Second, it may detect a model it can't handle via the 'sibling transitivity' test and return $\emptyset$. In this sense the algorithm also detects non-classical models without giving false positives. 


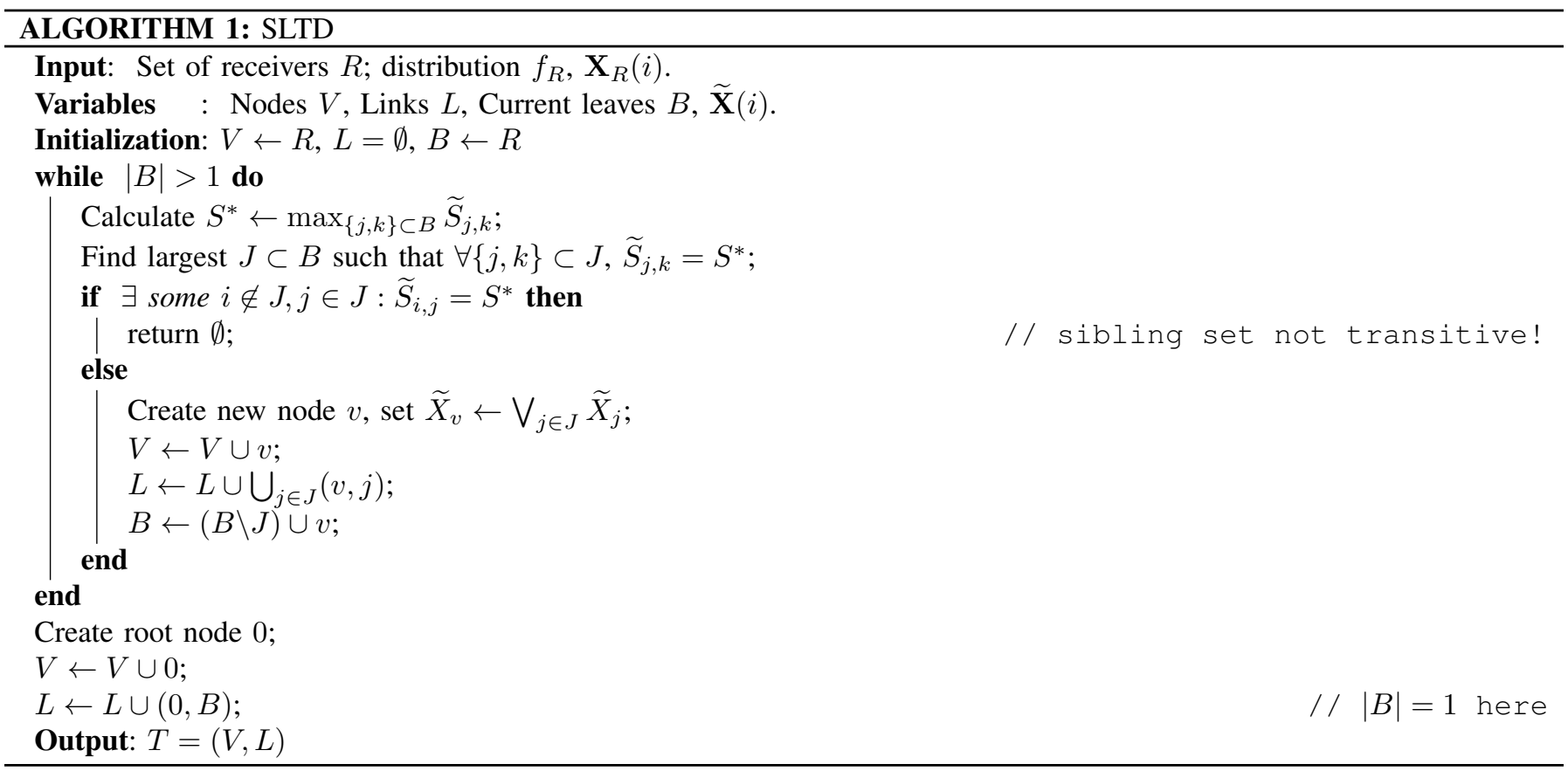

\section{Classically Equivalent Models}

We now present a simple means of introducing spatial dependencies that allows us to explore a much broader set of topologically determinate models.

Definition 2. (Classically Equivalent (CE) models)

$$
\mathcal{M}_{\mathrm{CE}}=\left\{M: \exists M^{\prime} \in \mathcal{M}_{\mathrm{C}} \text { with } f_{R}(M)=f_{R}\left(M^{\prime}\right) \text { and } T(M)=T\left(M^{\prime}\right)\right\} .
$$

That is, CE models are those for which a classical model exists with the same output distribution and topology.

Proposition 2. $\mathcal{M}_{\mathrm{CE}}$ is topologically determinate.

Proof. Let $M_{1}, M_{2} \in \mathcal{M}_{\mathrm{CE}}$ with $f_{R}\left(M_{1}\right)=f_{R}\left(M_{2}\right)$. By the definition of $\mathcal{M}_{\mathrm{CE}}, \exists M_{1}^{\prime} \in \mathcal{M}_{\mathrm{C}}$ such that $f_{R}\left(M_{1}^{\prime}\right)=f_{R}\left(M_{1}\right)$ and $T\left(M_{1}^{\prime}\right)=T\left(M_{1}\right)$. Similarly, $\exists M_{2}^{\prime} \in \mathcal{M}_{\mathrm{C}}$ such that $f_{R}\left(M_{2}^{\prime}\right)=f_{R}\left(M_{2}\right)=f_{R}\left(M_{1}\right)=f_{R}\left(M_{1}^{\prime}\right)$ and $T\left(M_{2}^{\prime}\right)=T\left(M_{2}\right)$. Since for any $f$ there is at most one $M \in \mathcal{M}_{\mathrm{C}}$ with $f_{R}(M)=f$ (Theorem 2) we have $M_{1}^{\prime}=M_{2}^{\prime}$ and so $T\left(M_{2}\right)=T\left(M_{2}^{\prime}\right)=$ $T\left(M_{1}^{\prime}\right)=T\left(M_{1}\right)$ as required.

Proposition 3. SLTD recovers $T(M)$ for all $M \in \mathcal{M}_{\mathrm{CE}}$.

Proof. By definition of $\mathcal{M}_{\mathrm{CE}}, \exists M^{\prime} \in \mathcal{M}_{\mathrm{C}}$ such that $f_{R}(M)=f_{R}\left(M^{\prime}\right)$. By Theorem 1 SLTD applied to $M^{\prime}$ recovers $T\left(M^{\prime}\right)$. The result follows from Proposition 1.

The significance of the $\mathcal{M}_{\mathrm{CE}}$ class is that it is the answer to the natural question of what non-trivial spatial dependencies are indistinguishable from classical ones from the receiver viewpoint, while retaining topological identifiability. It is the most natural generalization built 'around' the classical class $\mathcal{M}_{\mathrm{C}}$, and moreover is large (see Section IX). The significance of Proposition 3 is that it shows, for the first time, that topology recovery is feasible despite non-trivial spatial dependencies. The fact that SLTD was already known does not detract from this, as its non-classical properties have not been considered before.

The $\mathcal{M}_{\mathrm{CE}}$ definition is not constructive, making it difficult to 'see' the nature of the spatial dependencies allowed, and to check if they are consistent with the physics of data networks. Clearly, $\mathcal{M}_{\mathrm{CE}}$ is also constrained by its strong ties to classical models. To address this, we next seek a more explicitly parameterized class of topologically determinate models.

\section{JUMP INDEPENDENT MODELS}

In this section we introduce the Jump Independent (JI) model class, and give its main properties. The question of the topological determinism of JI models, and associated algorithms for topology recovery, is the topic of Section VIII. 


\section{A. JI Models: Definition and Fundamental Property}

We seek a model class honoring two principles, each abstracted from a knowledge of computer networks. The first is to allow local dependencies, that is between siblings at each node and their parent. This is because siblings map to output ports of a switching element, and their parent to an input port, across which dependencies can be naturally created through shared resources, such as router memory, and via shared loads as described in Section II. The second is to exclude 'action at a distance' dependencies. This is because we know of no normal mechanism in real networks which creates dependencies between non-adjacent links without affecting intermediate links in some way.

Definition 3 (Jump Independent Models). A model with nodes $V$ and receivers $R$ is Jump Independent if $\forall k \in V \backslash R, \mathbf{Z}_{c(k)}$ is conditionally mutually independent of $\mathbf{X}_{V \backslash(k \cup d(k))}$ given $X_{k}=1$.

In other words, when a probe reaches $k$, the loss behaviour over $k$ 's children is unaffected by what occurs outside of $k$, and vice versa.

It is not difficult to show that sufficiency of passage probabilities extends to statements of conditional independence. Thus the JI definition is equivalent to requiring

$$
\operatorname{Pr}\left(\bigcap_{i \in D}\left\{Z_{i}=1\right\} \mid X_{k}=1\right)=\operatorname{Pr}\left(\bigcap_{i \in D}\left\{Z_{i}=1\right\} \mid X_{k}=1, \bigcap_{j \in J}\left\{X_{j}=1\right\}\right)
$$

for any subset $D$ of $c(k)$, and subset $J$ obeying $J \cap d(k)=\emptyset$.

The above definition addresses the second principle by ruling out any dependencies not mediated in the natural way by intermediate links in the tree. Rather than conditioning on the value of the parent node $X_{k}$ in general, it is only necessary to condition on $X_{k}=1$, since if $X_{k}=0$ then $X_{i}=0$ for all $i \in d(k)$, regardless of the distribution of $\mathbf{Z}_{c(k)}$. The first principle is comprehensively addressed by allowing arbitrary dependencies over siblings, which is desirable since we do not know $a$ priori what forms these may take. The model also allows dependency, in fact to the maximum extent possible, between parent and child links. Moreover the definition also allows arbitrary spatial dependencies between sequences of successive links in any subpath, for example induced by shared congestion as discussed in Section II. Finally, there is no hidden assumption on temporal dependencies, for example an artificial assumption of temporal independence on each link, since this paper deals with the joint marginal distribution of the receiver observations only.

We now prove a powerful property of this class, $\mathcal{M}_{\mathrm{JI}}$, of Jump Independent models. Let $T=(V, L)$ be a tree, let $j \in V$ and let $D$ be a set of nodes with $D \subset c(j)$. For convenience we define $\rho_{D}=\operatorname{Pr}\left(\cap_{i \in D}\left\{Z_{i}=1\right\} \mid X_{j}=1\right)=\operatorname{Pr}\left(\cap_{i \in D}\left\{X_{i}=\right.\right.$ $\left.1\} \mid X_{j}=1\right)$.. We sometimes write $\rho_{i}$ and $\rho_{i, j}$ as shorthand versions of $\rho_{\{i\}}$ and $\rho_{\{i, j\}}$ respectively. These values give the probability of a probe progressing from the parent to its children. The following result shows that the probability of any probe transmission event over the whole tree can be expressed in terms of these local $\rho_{D}$ values.

For any arbitrary subset of nodes $U \subset V$ we define the subtree induced by $U$ as $T(U)=(v(U), \ell(U))$, the smallest (connected) subtree of $T$ that includes both the root and all the nodes in $U$, that is $v(U)=U \cup \bigcup_{k \in U} a(k)$ and $\ell(U)=$ $\bigcup_{k \in U}(0 \rightarrow k)$. Let $R(U)$ be the leaf nodes of $T(U)$, that is, those with no descendents in $T(U)$. Note that while $T(U)$ is a tree, it may have nodes with only one child, unlike $T$ itself, and $\bigcap_{j \in U}\left\{X_{j}=1\right\}=\bigcap_{j \in T(U)}\left\{X_{j}=1\right\}=\bigcap_{j \in T(U)}\left\{Z_{j}=1\right\}$.

Proposition 4 (Fundamental Property of JI Models). Let $M\left(T, f_{\mathbf{Z}}\right)$ be a Jump Independent model. Then for all $U \subset V$

$$
\operatorname{Pr}\left(\bigcap_{k \in U}\left\{X_{k}=1\right\}\right)=\prod_{i \in T(U) \backslash R(U)} \rho_{c(i) \cap T(U)} .
$$

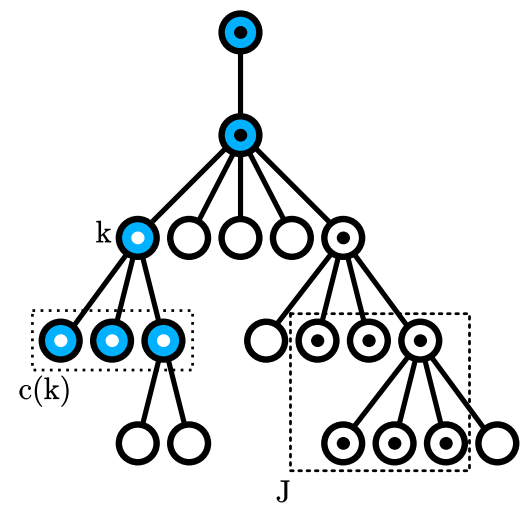

Fig. 3. Example of the conditioning from (4) following Definition 3. Shown are sets $D=c(k)$, and $J$ such that $J \cap d(k)=\emptyset$, together with the induced trees above them (resp. blue annuli and black dots) over which passage is required. 
Proposition 4 is powerful as it states that any passage probability factorizes in terms of local conditional probabilities. In particular, by setting $U \subset R$ it implies that $f_{R}$ can be expressed in this way.

Proof. Write nodes $T(U)$ in breadth-first ordering $\left(u_{1}, \ldots, u_{p}\right)$ starting from the root. This means that nodes are in order of increasing distance from the root, and siblings are adjacent in the the ordering. Partition $\left(u_{1}, \ldots, u_{p}\right)$ into groups of siblings, $\left(J_{1}, \ldots, J_{q}\right)$ while keeping the order. Then

$$
\begin{aligned}
& \operatorname{Pr}\left(\bigcap_{k \in U}\left\{X_{k}=1\right\}\right)=\operatorname{Pr}\left(\bigcap_{k \in T(U)}\left\{X_{k}=1\right\}\right) \\
& =\prod_{i=1, \ldots, q} \operatorname{Pr}\left(\bigcap_{k \in J_{i}}\left\{X_{k}=1\right\} \mid \bigcap_{k \in J_{1} \cup \ldots \cup J_{i-1}}\left\{X_{k}=1\right\}\right) \\
& =\prod_{i=1, \ldots, q} \operatorname{Pr}\left(\bigcap_{k \in J_{i}}\left\{Z_{k}=1\right\} \mid \bigcap_{k \in J_{1} \cup \ldots \cup J_{i-1}}\left\{X_{k}=1\right\}\right) \\
& =\prod_{i=1, \ldots, q} \operatorname{Pr}\left(\bigcap_{k \in J_{i}}\left\{Z_{k}=1\right\} \mid\left\{X_{f\left(J_{i}\right)}=1\right\}\right) \\
& =\prod_{i=1, \ldots, q} \rho_{J_{i}}
\end{aligned}
$$

where (6) follows from repeated application of the rule $\operatorname{Pr}(A \cap B)=\operatorname{Pr}(A \mid B) \operatorname{Pr}(B)$, (7) because $\left\{X_{k}=1\right\}$ is equivalent to $\left\{Z_{k}=1\right\} \cap\left\{X_{f(k)}=1\right\}$, and (8) is a direct application of the Jump Independence definition since the $J_{1}, \ldots, J_{i}$ are sibling sets in a breadth-first ordering, and so $J_{1} \cup \ldots \cup J_{i-1}$ does not intersect $d\left(f\left(J_{i}\right)\right)$. The $J_{1}, \ldots, J_{q}$ in $(9)$ are precisely the $c(i) \cap T(U)$ for $i \in T(U) \backslash R(U)$ in (5), so we are done.

Note that it is not difficult to show, using techniques similar to that of Proposition 4 , that the conditional independence of $\mathbf{Z}_{c(k)}$ and $\mathbf{X}_{V \backslash(k \cup d(k))}$ from the JI definition implies the same between $\mathbf{X}_{d(k)}$ and $\mathbf{X}_{V \backslash(k \cup d(k))}$. Thus an equivalent definition is conditional independence of loss over the inside and outside of the subtree rooted at $k$.

\section{B. Shared Loss/Transmission Properties}

The fundamental property allows us to evaluate the shared transmission $S_{i, j}$ for any two nodes in any JI model.

Corollary 1. Let $M=\left(T, f_{\mathbf{Z}}\right)$ be a Jump Independent model. Let $i, j \in V$ with shared path $0 \rightarrow b$ and $i \neq b \neq j$. Let $i^{\prime}=c(b) \cap(0 \rightarrow i)$ and $j^{\prime}=c(b) \cap(0 \rightarrow j)$

$$
S_{i, j}=\operatorname{Pr}\left(X_{b}=1\right) \cdot \frac{\rho_{i^{\prime}} \rho_{j^{\prime}}}{\rho_{i^{\prime}, j^{\prime}}}=\left(\prod_{k \in 0 \rightarrow b} \rho_{k}\right) \cdot \frac{\rho_{i^{\prime}} \rho_{j^{\prime}}}{\rho_{i^{\prime}, j^{\prime}}} .
$$

Proof. Follows by applying Proposition 4 to the definition (2) of $S_{j, k}$, setting $\rho_{\emptyset}=\rho_{i, \emptyset}=\rho_{\emptyset, \emptyset}=1$ as needed.

Corollary 1 means that the shared transmission consists of two parts in a JI model: the classical component $\operatorname{Pr}\left(X_{b}=1\right)$ for transmission along the physically shared path itself, and an extra factor incorporating the dependency between the two relevant children of the branch point, which vanishes in the classical case. Note $S_{i, j}=\operatorname{Pr}\left(X_{i}=1\right)$ if $j \in d(i)$ or $j=i$.

Just as in the classical case, the natural estimate for the (partially-observed) passage variables of an internal (or virtual during topology inference) node $v$ is given by $\widetilde{X}_{v}=\bigvee_{j \in c(v)} \widetilde{X}_{j}=\bigvee_{j \in R(v)} X_{j}$.

Lemma 2. The shared loss $\widetilde{S}_{j, k}$ calculated when using $\widetilde{X}_{j}$, $\widetilde{X}_{k}$ is equal to the actual shared loss $S_{j, k}$.

Proof. Express $\widetilde{X}_{j}$ as $\tilde{X}_{j}=X_{j} G_{j}$, where

$$
\begin{gathered}
G_{j}=\bigvee_{r \in R(j)} Y_{j \rightarrow r}=\bigvee_{r \in R(j)} \bigwedge_{i \in j \rightarrow r} Z_{i} . \\
\operatorname{Pr}\left(\widetilde{X}_{j}=1\right)=\operatorname{Pr}\left(X_{j}=1 \wedge G_{j}=1\right)=\operatorname{Pr}\left(X_{j}=1\right) \operatorname{Pr}\left(G_{j}=1 \mid X_{j}=1\right) .
\end{gathered}
$$

Let $j, k$ be nodes such that $d(j) \cap d(k)=\emptyset$, then

$$
\begin{aligned}
& \operatorname{Pr}\left(\left\{X_{j}=1\right\} \wedge\left\{G_{j}=1\right\}\right)=\operatorname{Pr}\left(\bigcup_{i \in R(j)}\left\{X_{i}=1\right\}\right) \\
& =\sum_{U \subseteq R(j)}(-1)^{|U|+1} \operatorname{Pr}\left(\bigcap_{i \in U}\left\{X_{i}=1\right\}\right) \\
& =\sum_{U \subseteq R(j)}(-1)^{|U|+1} \prod_{i \in T(U) \backslash R(U)} \operatorname{Pr}\left(\bigcap_{l \in c(i) \cap T(U)} Z_{l}=1 \mid X_{i}=1\right)
\end{aligned}
$$




$$
\begin{aligned}
& =\sum_{U \subseteq R(j)}(-1)^{|U|+1} \prod_{i \in a(j)} \operatorname{Pr}\left(\bigcap_{l \in c(i) \cap T(U)} Z_{l}=1 \mid X_{i}=1\right) \prod_{i \in T(U, j)} \operatorname{Pr}\left(\bigcap_{l \in c(i)} Z_{l}=1 \mid X_{i}=1\right) \\
& =\operatorname{Pr}\left(X_{j}=1\right) \sum_{U \subseteq R(j)}(-1)^{|U|+1} \prod_{i \in T(U, j)} \operatorname{Pr}\left(\bigcap_{l \in c(i) \cap T(U)} Z_{l}=1 \mid X_{i}=1\right)
\end{aligned}
$$

Here $T(U, j)$ represents $T(U) \cap(d(j) \cup j) \backslash R(U)$, so

$$
\begin{aligned}
\operatorname{Pr}\left(G_{j}=1 \mid X_{j}=1\right) & =\sum_{U \subseteq R(j)}(-1)^{|U|+1} \prod_{i \in T(U, j)} \operatorname{Pr}\left(\bigcap_{l \in c(i)} Z_{l}=1 \mid X_{i}=1\right) \\
& =\sum_{U \subseteq R(j)}(-1)^{|U|+1} \prod_{i \in T(U, j)} \operatorname{Pr}\left(\bigcap_{l \in c(i)} Z_{l}=1 \mid X_{i}=1, X_{k}=1\right) \\
& =\operatorname{Pr}\left(G_{j}=1 \mid X_{j}=1, X_{k}=1\right)
\end{aligned}
$$

from Jump Independence. Similarly, since $G_{j}$ and $G_{k}$ are determined by the probabilities $\operatorname{Pr}\left(\bigcap_{l \in c(i)} Z_{l}=1 \mid X_{i}=1\right)$ for nodes $i$ below $j, k$ respectively, then they will be conditionally independent given $X_{j}=1$ and $X_{k}=1$, so $\operatorname{Pr}\left(G_{k}=1 \mid X_{k}=1, X_{j}=\right.$ $\left.1, G_{j}=1\right)=\operatorname{Pr}\left(G_{k}=1 \mid X_{k}=1\right)$. Thus (in the following we write $\operatorname{Pr}\left(X_{j}\right)$ instead of $\operatorname{Pr}\left(X_{j}=1\right)$ ):

$$
\begin{aligned}
\widetilde{S}_{j, k} & =\frac{\operatorname{Pr}\left(\widetilde{X}_{j}\right) \operatorname{Pr}\left(\widetilde{X}_{k}\right)}{\operatorname{Pr}\left(\widetilde{X}_{j}, \widetilde{X}_{k}\right)}=\frac{\operatorname{Pr}\left(X_{j}, G_{j}\right) \operatorname{Pr}\left(X_{k}, G_{k}\right)}{\operatorname{Pr}\left(X_{j}, G_{j}, X_{k}, G_{k}\right)}=\frac{\operatorname{Pr}\left(X_{j}\right) \operatorname{Pr}\left(G_{j} \mid X_{j}\right) \operatorname{Pr}\left(X_{k}\right) \operatorname{Pr}\left(G_{k} \mid X_{k}\right)}{\operatorname{Pr}\left(X_{j}, X_{k}\right) \operatorname{Pr}\left(G_{j} \mid X_{j}, X_{k}\right) \operatorname{Pr}\left(G_{k} \mid X_{j}, X_{k}, G_{j}\right)} \\
& =\frac{\operatorname{Pr}\left(X_{j}\right) \operatorname{Pr}\left(G_{j} \mid X_{j}\right) \operatorname{Pr}\left(X_{k}\right) \operatorname{Pr}\left(G_{k} \mid X_{k}\right)}{\operatorname{Pr}\left(X_{j}, X_{k}\right) \operatorname{Pr}\left(G_{j} \mid X_{j}\right) \operatorname{Pr}\left(G_{k} \mid X_{k}\right)}=S_{j, k}
\end{aligned}
$$

\section{JI models that are Classically Equivalent}

We are motivated to examine models in $\mathrm{CE} \cap \mathrm{JI}$ in order to gain a concrete understanding of a physically meaningful component of the CE class, and conversely, to see how JI can step beyond CE.

Let $M\left(T, f_{\mathbf{Z}}\right)$ be a jump independent model such that $T$ is strictly binary. Let $s(i)$ be the unique sibling of $i$, and $c_{1}(i)$ and $c_{2}(i)$ be the children of $i$ (in lexicographic order without loss of generality). Then set

$$
l_{i}= \begin{cases}\frac{\rho_{i, s(i)}}{\rho_{s(i)}}, & \text { if } i \in R \\ \rho_{1} \cdot \frac{\rho_{c_{1}(1)} \rho_{c_{2}(1)}}{\rho_{c_{1}(1), c_{2}(1)}} & \text { if } i=1 \\ \frac{\rho_{i, s(i)}}{\rho_{s(i)}} \cdot \frac{\rho_{c_{1}(i)} \rho_{c_{2}(i)}}{\rho_{c_{1}(i), c_{2}(i)}} & \text { otherwise. }\end{cases}
$$

Theorem 3. Let $M \in \mathcal{M}_{\mathrm{JI}}$ have topology $T=(V, L)$ that is strictly binary. Let $M^{\prime}\left(T, f_{\mathbf{Z}}^{\prime}\right) \in \mathcal{M}_{C}$ have the same topology with $\operatorname{Pr}\left(Z_{i}=1\right)=l_{i}$ defined by (11). If $0<l_{i}<1$ for all $i \in L$ then $f_{R}(M)=f_{R}\left(M^{\prime}\right)$ and thus $M \in \mathcal{M}_{\mathrm{CE}}$.

This result (see Appendix for a proof) shows that any binary jump independent model is measurement equivalent to a classical model provided $f_{\mathbf{Z}}$ satisfies a set of simple local conditions, corresponding to the requirement that no pair of sibling link variables are so negatively correlated that this correlation cannot be reallocated to their ancestor links by adjusting their loss probabilities within $(0,1)$. When $0<l_{i}<1$ does not hold for all links, the JI model then provides (as we will show) an explicit example of topologically determinate models outside of $\mathcal{M}_{\mathrm{CE}}$. It also leads to insights into the relative sizes of these classes, as discussed in Section IX-C.

\section{A SLTD ALGORITHM FOR JI MODELS}

We introduce Shared Loss Topology Discovery 2 (SLTD2), a topology inference algorithm for JI models. Like SLTD, it is based on looking at shared loss $S_{i, j}$ across pairs of receivers, but in a very different way. We prove that it can recover the topology of 'almost all' JI models, including all those with binary topologies, and all classical models.

We define a sibling set to mean all the children of a single node, whereas a set of siblings means any subset of such. An available set consists of the members of a sibling set that are receivers, and if they all are, we have an available sibling set.

For classical models Certain Paternity (Lemma 1) enabled the key step of the topology recovery algorithm: the ability to identify an available sibling set. Here the situation is more complex and several preliminary properties are required. We establish these in Section VIII-A before moving to the algorithm itself in Section VIII-B, and a discussion of the topological determinism of JI models in Section VIII-C. 

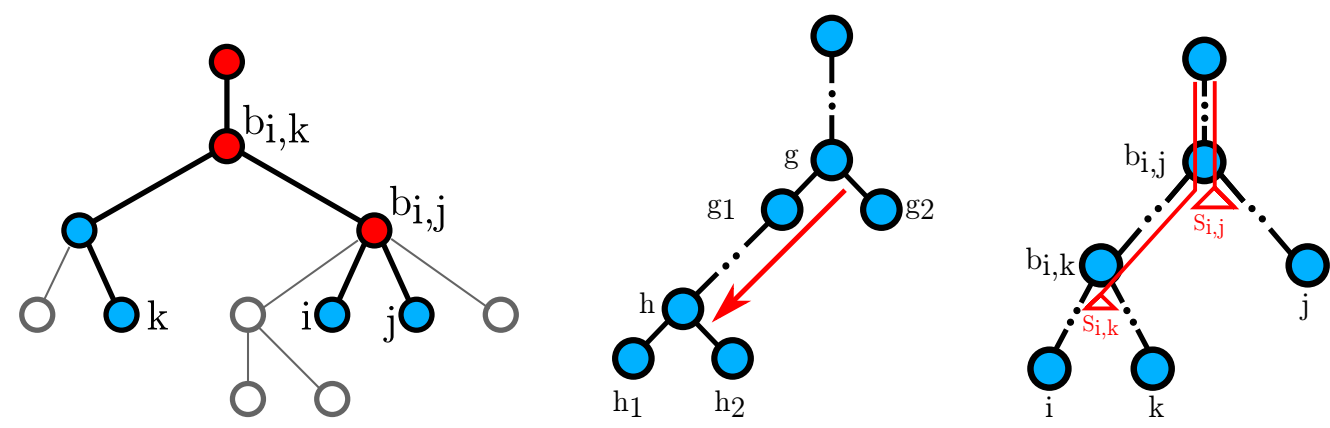

Fig. 4. Left: siblings share the same 'world view'; the same shared loss to nodes outside their parent's descendants, thus nodes $i, j$ have the same shared loss to $k$. Centre: The augmented subpath $g\left(g_{1}, g_{2}\right) \rightarrow h\left(h_{1}, h_{2}\right)$. Right: invisible subpaths (Proposition 5). In this situation where $k$ is not 'outside of' $b_{i, j}$, $S_{i, k}$ cannot equal $S_{j, k}$ unless the subpath from $b_{i, j}$ to $b_{i, k}$ is invisible. Half-dotted lines denote one or more links in series.

\section{A. Finding Sibling Sets: Certain Paternity for JI Models}

A basic property underlying certain paternity for classical models is that $S_{i, j}=\operatorname{Pr}\left(X_{b_{i, j}}=1\right)$ is a constant for any pair $(i, j)$ of siblings from some sibling set. This form of 'internal agreement' property will not hold in general for non-classical models. Further, the shared transmission value will no longer necessarily decrease as the shared path lengthens, another property on which SLTD was based. We now give a complementary sibling property based on 'external agreement', which holds for all JI models, both classically equivalent and not.

Lemma 3 (Siblings Agree Externally). Let $M \in \mathcal{M}_{\mathrm{JI}}$. If two nodes $i, j$ are members of a sibling set $J$, then for all $k \in R \backslash d(f(J)), S_{i, k}=S_{j, k}$.

Proof. Follows immediately from Corollary 1.

Thus siblings agree on the shared loss seen between themselves and any nodes outside the 'family'; for example $S_{i, k}=S_{j, k}$ on the left in Figure 4. We seek to invert this relationship in order to find available sibling sets through a new form of certain paternity. To this end define the agreement set of a pair $\{i, j\}$ of nodes as

$$
A_{i, j}=\left\{k \in R: S_{i, k}=S_{j, k}, k \neq i, j\right\} .
$$

The set $A_{i, j}$ includes all receivers outside the subtree rooted at the branch node $b_{i, j}$, but may also include nodes lying under $b_{i, j}$. Dealing with this latter possibility requires that restrictions be imposed, and different cases distinguished. Without these restrictions an erroneous topology could be inferred that is missing some internal nodes.

An augmented subpath $g\left(g_{1}, g_{2}\right) \rightarrow h\left(h_{1}, h_{2}\right)$ is a path $g \rightarrow h$ together with $g_{1}, g_{2} \in c(g), h_{1}, h_{2} \in c(h)$ such that $g_{1} \in g \rightarrow h$ (see Figure 4 centre; recall that $g \rightarrow h$ does not include the link/node $g$ itself). We call such an augmented subpath invisible if

$$
\frac{\rho_{g_{1}} \rho_{g_{2}}}{\rho_{g_{1}, g_{2}}}=\left(\prod_{i \in g \rightarrow h} \rho_{i}\right) \frac{\rho_{h_{1}} \rho_{h_{2}}}{\rho_{h_{1}, h_{2}}} .
$$

If $g\left(g_{1}, g_{2}\right) \rightarrow h\left(h_{1}, h_{2}\right)$ is an invisible augmented subpath, for convenience we call the path $g \rightarrow h$ an invisible subpath.

Proposition 5. Let $i, j, k$ be three distinct receivers in a Jump Independent model such that $b_{i, k}$ is a descendant of $b_{i, j}$. Then $S_{i, k}=S_{j, k}$ if and only if $b_{i, j} \rightarrow b_{i, k}$ is invisible. Consequently, with no invisible subpaths, $k \in A_{i j}$ implies $b_{i, k} \notin d\left(b_{i, j}\right)$.

Proof. Follows immediately from the definitions of shared transmission and (10) (see rightmost plot in Figure 4).

The invisible subpath condition is the JI analogue of the classical requirement to avoid degenerate $l_{k}=1$ link passage probabilities which make the topology unidentifiable. If there are no invisible subpaths, $\left|A_{i, j}\right|$ is analogous to the shared loss in the classical tree, in that it increases as the branching point moves down a path away from the root. For fixed $i$ the size of $A_{i, j}$ increases as $j$ approaches $i$. Thus if $b_{i, j}$ is an ancestor of $b_{k, l}$, then $A_{i, j} \subset A_{k, l}$ and so $\left|A_{i, j}\right| \leq\left|A_{k, l}\right|$.

Proposition 5 removes an important source of ambiguity, but in order to identify available sets we must also place limits on the joint-distributions of sibling sets.

We say a sibling set $J$ is internally agreeing if $S_{i, j}=S_{k, l}$ for all $i, j, k, l \in J$ with $i \neq j, k \neq l$. We say a sibling set $J$ is internally disagreeing if $S_{i, j} \neq S_{k, l}$ for all $i, j, k, l \in J$ with $\{i, j\} \neq\{k, l\}$. Since the component of shared loss down to the branch node is identical for all siblings, the above are restrictions on the allowed form of (conditional) sibling dependency, and are therefore local in character.

Definition 4 (Agreeable JI models $\left(\mathcal{M}_{\mathrm{AJI}}\right)$ ). An AJI model is a model $M \in \mathcal{M}_{\mathrm{JI}}$ which satisfies the following conditions: i) (internally consistent) Each sibling set $J$ is internally agreeing or internally disagreeing.

ii) (no invisible subpaths) No augmented subpaths in $M$ are invisible. 


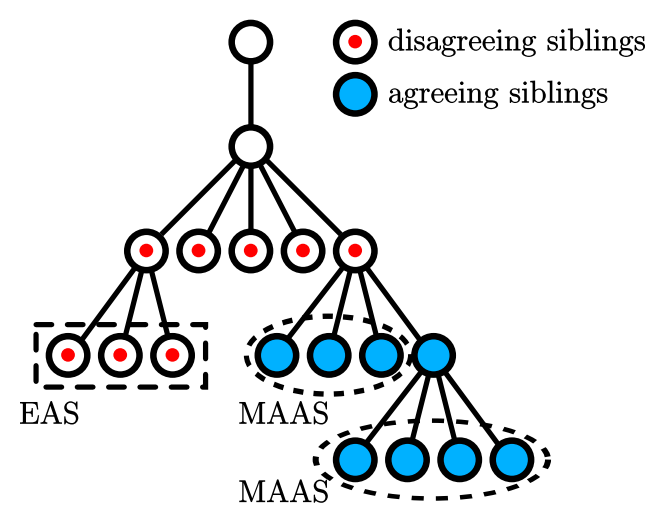

Fig. 5. An $\mathcal{M}_{\text {AJI }}$ model with EASes and MAASes displayed. The top group of siblings is not an EAS because it has siblings that aren't receivers. The higher of the two MAASes is not a sibling set either, but it is still an MAAS. In this case, the sibling set chosen in the proof of Proposition 6 is the EAS.

The motivation for this definition is that $\mathcal{M}_{\mathrm{AJI}}$, as we show below, is a Topologically Determinate class, whereas $\mathcal{M}_{\mathrm{JI}}$ itself is not. Each of conditions (i) and (ii) eliminate different ways in which identifiability can fail. Condition (i) has two components. Internally disagreeing siblings is the generic case with maximal dimension in the parameter space. Internally agreeing siblings is a special case of lower dimension, but it is important as it includes classical models. Together, these make $\mathcal{M}_{\text {AJI }}$ a large subset of $\mathcal{M}_{\text {JI }}$ which is both identifiable as well as a generalization of classical models. For binary topologies (i) is trivially satisfied.

We now set out to prove that $\mathcal{M}_{\mathrm{AJI}}$ is topologically determinate (Theorem 4). We do this by working towards an algorithm capable of recovering the topology, focussing on the key problem of correctly identifying sibling sets.

Definition 5 (All-agreeing sets (AAS)). Call $D \subset R$ with $|D| \geq 2$ an all-agreeing set if $A_{i, j}=R \backslash\{i, j\}$ for all $i, j \in D$. Any subset of an all-agreeing set is also an all-agreeing set. We call an all-agreeing set $D$ a maximal all-agreeing set (MAAS) if it is not a proper subset of another all-agreeing set.

Definition 6 (Externally-agreeing sets (EAS)). Call $D \subset R$ an externally-agreeing set (EAS) if $|D| \geq 3$ and $A_{i, j}=R \backslash D$ for all $i, j \in D$.

The AAS and EAS can be determined from the pairwise shared loss values for nodes in $R$. We can find the internallydisagreeing available sibling sets (IDASS) using the EAS, and the internally-agreeing available sets (IAAS) using the MAAS. The latter are not guaranteed to be full sibling sets (they might have another sibling that is not a receiver, see Figure 5), so we may need an extra step to choose one that is. The next two lemmas have proofs in the Appendix.

Lemma 4 (Finding internally-agreeing available sets (IAAS)). Consider an AJI model with receivers $R$. A set $D \subset R$ with $|D| \geq 2$ is an IAAS if and only if it is a MAAS.

Consequently, finding the MAAS allows us to find the IAAS of size at least 2. Among these will be all the internally agreeing available sibling sets.

Lemma 5 (Finding internally-disagreeing available sibling sets (IDASS)). Consider an AJI model with receivers $R$. A set $D \subset R$ with $|D| \geq 3$ is an IDASS if and only if it is an EAS.

This result is more powerful than Lemma 4 in that every set it identifies is a sibling set, rather than just an available set. Further, it identifies all the available sibling sets of disagreeing type.

We are at last able to give the paternity result which forms the basis of the SLTD2 algorithm.

Proposition 6 (Certain Paternity II). Assume $M \in \mathcal{M}_{\mathrm{AJI}}$. Then at least one available sibling set can be identified without error.

Proof. The shared transmissions $S_{i, j}$ can be calculated for all pairs $\{i, j\} \in R$. From these, all of the MAAS and all the EAS can be determined, and therefore all the IAAS (Lemma 4), and all the IDASS (Lemma 5), can be found. The union of these cannot be empty, since the tree must have at least one available sibling set, which by the AJI assumption, must be either internally agreeing or internally disagreeing.

If any IDASS is found then the lemma is proved (see Figure 5). If not, then there is at least one IAAS and we must find one which is a sibling set. Consider an IAAS $D$ which is not a sibling set (if there is no such $D$ then any of the IAAS will do). Then there exists a sibling $k$ of the nodes in $D, k \notin R$, which will be an ancestor of at least one other IAAS, say $D^{\prime}$. To detect that $D^{\prime}$ is indeed below $D$, take an $i \in D$ and $j \in D^{\prime}$ and form $A_{i, j}$. Then $A_{i, j} \cap D^{\prime}=\emptyset$, but $A_{i, j} \cap D=D \backslash i$. If instead $D^{\prime}$ is in another branch, both intersections are null. It is obvious that an IAAS is a sibling set iff there are no other 
IAAS below it. Clearly, by using the above test over all pairs of IAAS all such sets can be found, any one of which will satisfy the lemma.

\section{B. SLTD2}

Just as in SLTD, SLTD2 works from the bottom up to reconstruct the topology. The algorithm maintain a set of nodes $B$ that have unknown parents with the idea that these correspond to receivers of an unknown subtree $V=T(B)$ of the real tree $T$. At each iteration, using certain paternity SLTD2 will find an $E \subset B$ that corresponds to an available sibling set from $V$. A virtual node $v$ and its associated inferred passage variable $\widetilde{X}_{v}$ will be created as a parent for these siblings, and $B$ will be updated by removing $E$ and adding $v$ in its place.

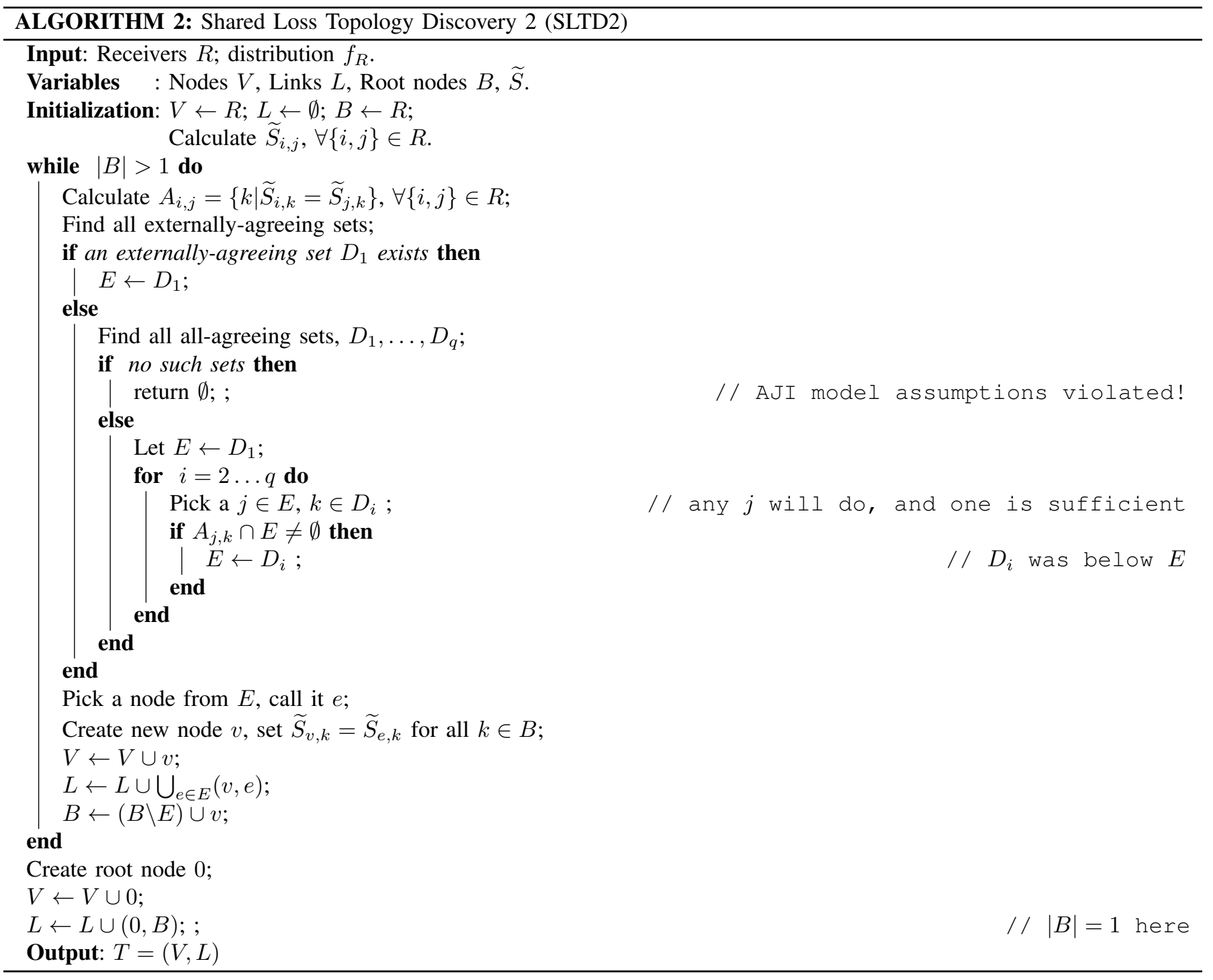

Theorem 4 (Correctness of SLTD2 on $\left.\mathcal{M}_{\mathrm{AJI}}\right)$. Let $M=\left(T, f_{\mathbf{Z}}\right)$ be in $\mathcal{M}_{\mathrm{AJI}}$. Then SLTD2 returns $T$.

Proof. If $M \in \mathcal{M}_{\mathrm{AJI}}$ then certain paternity II holds at the first iteration as all nodes are receivers and $\widetilde{S}_{i, j}=S_{i, j}$. The algorithm steps then echo the proof of Proposition 6. Note that after the candidate $E$ is updated in the AAS code branch the search can continue without reinitializing $i$, since $E$ must already be below all $D_{i}$ checked so far. Upon exit of the for loop $E$ is an AAS which has no other set below it as required.

Subsequent interations involve $\widetilde{S}_{i, j}$, but from Lemma $2 \widetilde{S}_{i, j}=S_{i, j}$, so any pair of virtual nodes $\widetilde{v}_{1}$, $\widetilde{v}_{2}$ will have the same shared transmission as the pair of real nodes they represent. Thus, at each step $\widetilde{S}_{i, j}$ will be correct provided the previous step was correct. The result then follows by induction.

For some $M \notin \mathcal{M}_{\mathrm{AJI}}$ it may happen that there are no externally-agreeing or all-agreeing sets. In that case the algorithm exits with an error which correctly indicates that the input model was not in $\mathcal{M}_{\mathrm{AJI}}$. 


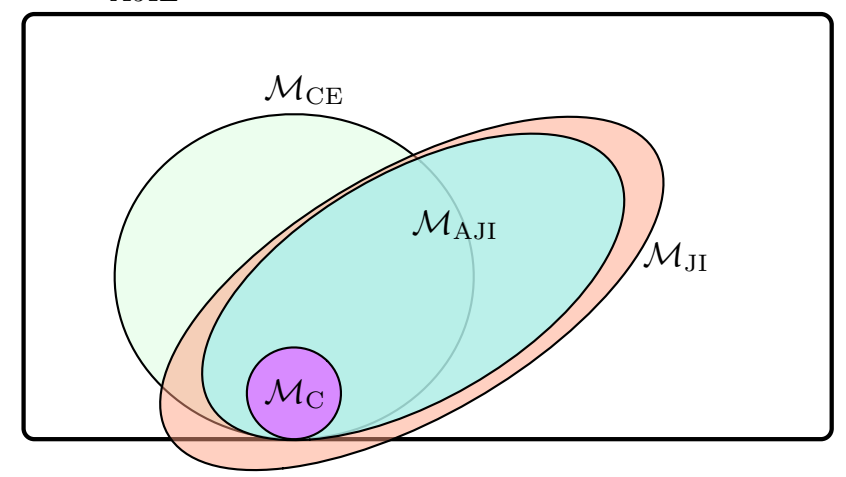

Fig. 6. Relationship between the model classes. Note that $\mathcal{M}_{\mathrm{C}} \subset \mathcal{M}_{\mathrm{CE}} \subset \mathcal{M}_{\mathrm{AJIE}}$, and $\mathcal{M}_{\mathrm{C}} \subset \mathcal{M}_{\mathrm{AJI}} \subset \mathcal{M}_{\mathrm{AJIE}}$.

If used on an $M \notin \mathcal{M}_{\mathrm{AJI}}$ the algorithm may return an incorrect topology. It may be possible in some cases to analyse the detailed algorithm outputs to determine that they are inconsistent, and hence to detect that $M \notin \mathcal{M}_{\mathrm{AJI}}$.

\section{C. $\mathcal{M}_{\mathrm{AJIE}}$ : a Large Topologically Determinate Class}

We have already indicated that $\mathcal{M}_{\mathrm{JI}}$ is not topologically determinate because it includes models with invisible subpaths. A disturbing consequence is that, for any observable distribution $f_{R}$ on receivers, the true topology is indistinguishable from the degenerate 'receiver tree' topology, also in $\mathcal{M}_{\mathrm{JI}}$, where all receivers have the same father.

On a brighter note, Theorem 4 implies that $\mathcal{M}_{\mathrm{AJI}}$ is topologically determinate, and this is significant because $\mathcal{M}_{\mathrm{AJI}}$ covers 'almost all' of $\mathcal{M}_{\mathrm{JI}}$, due to the inclusion of the internally disagreeing subclass of JI models within $\mathcal{M}_{\mathrm{AJI}}$. This subclass has maximal dimension in $\mathcal{M}_{\mathrm{JI}}$, as we discuss in the next section.

To complete our analysis of the JI class and topological determinism, we extend $\mathcal{M}_{\mathrm{AJI}}$ to form the set of AJI equivalent models, $\mathcal{M}_{\mathrm{AJIE}}$, in essentially the same way that we extended $\mathcal{M}_{\mathrm{C}}$ to form $\mathcal{M}_{\mathrm{CE}}$.

Definition 7. (AJI Equivalent models)

$$
\mathcal{M}_{\mathrm{AJIE}}=\left\{M: \exists M^{\prime} \in \mathcal{M}_{\mathrm{JI}} \text { with } f_{R}(M)=f_{R}\left(M^{\prime}\right) \text { and } T(M)=T\left(M^{\prime}\right)\right\} .
$$

In the same way as before, this extension is topologically determinate, and Proposition 1 implies that SLTD2 applied to any $M \in \mathcal{M}_{\mathrm{AJIE}}$ will correctly recover $T(M)$.

Since $\mathcal{M}_{\mathrm{C}} \subset \mathcal{M}_{\mathrm{AJI}}$, it follows trivially that $\mathcal{M}_{\mathrm{CE}} \subset \mathcal{M}_{\mathrm{AJIE}}$. This shows the importance of including the internally agreeing subclass (and hence classical models) in the definition of $\mathcal{M}_{\mathrm{AJI}}$, even though the subclass is insignificant in a dimensional sense with respect to $\mathcal{M}_{\text {AJI }}$.

To conclude, as illustrated in Figure 6, we have found a topologically determinate class, $\mathcal{M}_{\mathrm{AJIE}}$, which includes all models from the other topologically determinate classes we have found, $\mathcal{M}_{\mathrm{CE}}$ and $\mathcal{M}_{\mathrm{AJI}}$, and have provided an associated topology recovery algorithm, SLTD2. By construction, $\mathcal{M}_{\mathrm{AJIE}}$ is the most natural and broadest generalization build 'around' $\mathcal{M}_{\mathrm{AJI}}$ which is still topologically determinate. As Figure 6 suggests, it represents the inclusion of many models in $\mathcal{M}_{\mathrm{JI}} \backslash \mathcal{M}_{\mathrm{AJI}}$ as well as outside of $\mathcal{M}_{\mathrm{JI}}$.

\section{The Relative sizes of C, CE, JI, AJI, AJIE.}

In this section we inquire whether $\mathcal{M}_{\mathrm{CE}}$ and $\mathcal{M}_{\mathrm{JI}}$ are large in some well defined sense, and thereby explore the relationships between these and related model classes.

We begin with some definitions. Let $\mathcal{M}_{T}$ denote the space of all possible models with topology $T, \mathcal{F}_{m}$ is the set of joint distributions on $m$ binomial variables, and $\mathcal{T}_{m}$ the set of tree topologies with $m$ receivers. Let $\mathcal{F}=\cup_{m \in \mathbb{N}} \mathcal{F}_{m}$.

Define a function $\phi: \mathcal{M} \rightarrow \mathcal{F}$ by $\phi(M)=f_{R}(M)$, mapping models to their observable receiver distributions. Note that $\phi$ is many-to-one: many different models sharing the correct $m$ can give the same receiver output. The set of models that are measurement equivalent to some $M$ can be written as $\phi^{-1}(\phi(M))=\left\{M^{\prime}: \phi\left(M^{\prime}\right)=\phi(M)\right\}$.

\section{A. Parameterizations}

To establish a notion of comparative size we compare the dimensions of the models' parameter spaces. To do so we must fix the topology under consideration, since the number of parameters required to characterize a model of a given class is topology dependent. Let $T=(V, L) \in \mathcal{T}_{m}$ have $n$ links. We can view a model $M \in \mathcal{M}_{T}$ as a vector $\mathbf{e}=\left(e_{1}, \ldots, e_{d}\right) \in[0,1]^{d}$ using 
the elementary probabilities parameterization, where $d=2^{n}-1$ is the dimensionality of the underlying parameter space (see Section III).

Specifically, for each $j \in\{1,2, \ldots, d\}$ define $\mathcal{I}(j) \subset\{1,2, \ldots, n\}$ as the unique subset of links such that $\sum_{i \in \mathcal{I}(j)} 2^{i}=j$. Then set

$$
e_{j}=\operatorname{Pr}\left(\bigcap_{i \in \mathcal{I}(j)}\left\{Z_{i}=1\right\} \cap \bigcap_{i \in L \backslash \mathcal{I}(j)}\left\{Z_{i}=0\right\}\right)
$$

as the $j$-th parameter. That is, we map the parameter label $j$ to its link passage pattern as follows: take the binary expansion ( $n$ bits long) of $j$, and in the places corresponding to non-zero bits, require passage over the corresponding (based on their arbitrary labels) links, and loss over the other links. Note that a vector e represents a model iff $\mathbf{e} \in(0,1)^{d}$ and $\sum \mathbf{e}<1$.

If we parameterize the space $\mathcal{F}_{m}$ of the observables similarly, then we see that $\phi$ is a linear map between these two vector spaces, since each element of the vector corresponding to $\phi(\mathbf{e})$ is a sum of elements from $\mathbf{e}$.

We can also parameterize the link distribution $f_{\mathbf{Z}}$ of $M$ using the link passage probabilities: For $j=1, \ldots, d$ let

$$
p_{j}=\operatorname{Pr}\left(\bigcap_{i \in \mathcal{I}(j)}\left\{Z_{i}=1\right\}\right)
$$

and so $\mathbf{p}=\left(p_{1}, \ldots, p_{d}\right)$ parameterizes $M$ (see Section III).

The relationship between $\mathbf{p}$ and $\mathbf{e}$ for a model $M$ is $p_{j}=\sum_{k: \mathcal{I}(j) \subset \mathcal{I}(k)} e_{k}$. Consequently we can write this in matrix form as $\mathbf{p}=A_{T} \mathbf{e}$ where $A_{T}$ is a $d$ by $d$ matrix with the $(j, k)$-th element

$$
A_{T}(j, k)= \begin{cases}1 & \text { if } \mathcal{I}(j) \subset \mathcal{I}(k) \\ 0 & \text { otherwise. }\end{cases}
$$

This gives a way to check if a given parameter vector $\mathbf{p}$ corresponds to a set of joint passage probabilities of a valid model: we can check that the corresponding $\mathbf{e}$ is valid, i.e., that $A_{T}^{-1} \mathbf{p} \in(0,1)^{d}$ and $\sum\left(A_{T}^{-1} \mathbf{p}\right)<1$.

In a similar way we can parameterize the observable joint distribution $\mathbf{p}_{R} \in \mathcal{F}_{m}$, where $\mathbf{p}_{R}=f_{R}((\mathbf{p}, T))=\phi((\mathbf{p}, T))$, via an integer $k \in\left\{1, \ldots, d_{R}\right\}, d_{R}=2^{m}-1$, labelling receiver (path) passage events and their probabilities. Passage to a set of receivers $R_{1} \subset R$ is equivalent to passage on each link in $T\left(R_{1}\right)$. Each element of $\mathbf{p}_{R}$ is a probability $\operatorname{Pr}\left(\cap_{i \in U}\left\{X_{i}=1\right\}\right)$ for some $U \in R$ and thus is the same as the element of $\mathbf{p}$ that gives the probability $\operatorname{Pr}\left(\cap_{i \in T(U)}\left\{Z_{i}=1\right\}\right)$. Consequently, $\mathbf{p}_{R}=B_{T} \mathbf{p}$, where $B_{T}$ is a $d_{r} \times d$ binary matrix with a single 1 in each row, determined solely by the topology $T$.

\section{B. Dimensions}

Using the above parameterizations we can determine the dimensions of $\mathcal{M}_{\mathrm{C}, \mathrm{T}}, \mathcal{M}_{\mathrm{CE}, \mathrm{T}}, \mathcal{M}_{\mathrm{JI}, \mathrm{T}}, \mathcal{M}_{\mathrm{AJI}, \mathrm{T}}$ and $\mathcal{M}_{\mathrm{AJIE}, \mathrm{T}}$ and the spaces of output distributions $\phi\left(\mathcal{M}_{\mathrm{C}, \mathrm{T}}\right), \phi\left(\mathcal{M}_{\mathrm{CE}, \mathrm{T}}\right), \phi\left(\mathcal{M}_{\mathrm{JI}, \mathrm{T}}\right), \phi\left(\mathcal{M}_{\mathrm{AJI}, \mathrm{T}}\right)$ and $\phi\left(\mathcal{M}_{\mathrm{AJIE}, \mathrm{T}}\right)$ induced by each model class.

We start by determining the dimensionality $\operatorname{dim}\left(\mathcal{M}_{C}\right)$ of $\mathcal{M}_{C}$. We can specify a link distribution of $M \in \mathcal{M}_{C}$ by specifying $l_{1}, l_{2}, \ldots, l_{n}$, since for classical models $\mathbf{p}_{j}=\operatorname{Pr}\left(\bigcap_{i \in \mathcal{I}(j)}\left\{Z_{i}=1\right\}\right)=\prod_{i \in \mathcal{I}(j)} l_{i}$ from the independence property, hence $\operatorname{dim}\left(\mathcal{M}_{\mathrm{C}, \mathrm{T}}\right)=n$. Also, since SLTD provides a bijection between $\mathcal{M}_{\mathrm{C}, \mathrm{T}}$ and $\phi\left(\mathcal{M}_{\mathrm{C}, \mathrm{T}}\right)$ we have that

$$
\operatorname{dim}\left(\phi\left(\mathcal{M}_{\mathrm{C}, \mathrm{T}}\right)\right)=\operatorname{dim}\left(\mathcal{M}_{\mathrm{C}, \mathrm{T}}\right)=n .
$$

Now consider $\mathcal{M}_{\mathrm{CE}, \mathrm{T}}$. We know that $\phi\left(\mathcal{M}_{\mathrm{CE}, \mathrm{T}}\right)=\phi\left(\mathcal{M}_{\mathrm{C}, \mathrm{T}}\right)$, so

$$
\operatorname{dim}\left(\phi\left(\mathcal{M}_{\mathrm{CE}, \mathrm{T}}\right)\right)=\operatorname{dim}\left(\phi\left(\mathcal{M}_{\mathrm{C}, \mathrm{T}}\right)\right)=n .
$$

Recall $\mathbf{p}_{R}=\phi(\mathbf{p})$ consists simply of $d_{R}$ particular elements of $\mathbf{p}$. These are required to lie in an $n$-dimensional subset of $\mathbb{R}^{d_{R}}$, and the other $d-d_{R}$ elements of $\mathbf{p}$ are free to be anything (subject to the validity constraints). Thus

$$
\operatorname{dim}\left(\mathcal{M}_{\mathrm{CE}}\right)=n+d-d_{R}=n+2^{n}-2^{m} .
$$

Determining $\operatorname{dim}\left(\mathcal{M}_{\mathrm{JI}, \mathrm{T}}\right)$ is more difficult, since it is dependent on the topology. The task is to determine the number of parameters required to specify the full link distribution $f_{\mathbf{Z}}$ of a model $M \in \mathcal{M}_{\mathrm{JI}}$. We proceed by splitting this into the parameters required to specify $f_{\mathbf{X}}$, and those required to specify the remainder of $f_{\mathbf{Z}}$. To specify $f_{\mathbf{X}}$ it is only necessary to consider events of the form $\cap_{v \in V} X_{v}=1$ (and hence equivalent events of the form $\cap_{v \in T(V)} Z_{v}=1$, corresponding to particular elements of the $\mathbf{p}$ vector). Since each model is Jump Independent, specifying these probabilities is equivalent to specifying the $\rho$-values, that is, the values $\rho_{D}=\operatorname{Pr}\left(\cap_{i \in D} Z_{i}=1 \mid X_{f(D)}=1\right)$ for each set of siblings $D$. The $\rho$-values specify $f_{\mathbf{X}}$ by Proposition 4 . In the reverse direction, $f_{\mathbf{X}}$ specifies the $\rho$-values since $\operatorname{Pr}\left(\cap_{i \in D} Z_{i}=1 \mid X_{f(D)}=1\right)=\operatorname{Pr}\left(\cap_{i \in D} X_{i}=1\right) / \operatorname{Pr}\left(X_{f(D)}=1\right)$.

The $\rho$-values are a minimal specification of $f_{\mathbf{X}}$, since no $\rho$-value can be determined from the set of the other $\rho$-values for a given topology. The number of such $\rho$-values is

$$
N_{\rho}=\sum_{i \in T \backslash R}\left(2^{|c(i)|}-1\right) .
$$




\begin{tabular}{|c|c|c|c|c|c|c|c|c|}
\hline$(n, m)$ & $\begin{array}{l}(3,2) \\
\text { binary }\end{array}$ & $\begin{array}{l}(4,3) \\
-\end{array}$ & $\begin{array}{l}(6,4) \\
-\end{array}$ & $\begin{array}{l}(9,5) \\
\text { binary }\end{array}$ & $\begin{array}{l}9 \\
8000000 \\
8 \\
000 \\
(15,8) \\
\text { binary }\end{array}$ & $\begin{array}{l}\text { id } \\
\text { odooo } \\
\text { odoboobo } \\
\\
(14,11) \\
-\end{array}$ & $\begin{array}{c}\text { 19 } \\
\text { od odo } \\
\text { odo } \\
(14,10) \\
-\end{array}$ & 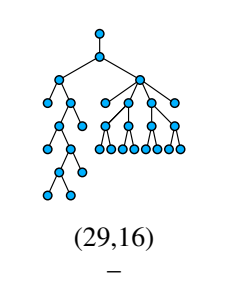 \\
\hline $\operatorname{dim}\left(\mathcal{M}_{\mathrm{C}, \mathrm{T}}\right), \quad \operatorname{dim}(\phi)$ & 3,3 & 4,4 & 6,6 & 9,9 & 15,15 & 14,14 & 14,14 & 29,29 \\
\hline $\operatorname{dim}\left(\mathcal{M}_{\mathrm{CE}, \mathrm{T}}\right), \quad \operatorname{dim}(\phi)$ & 7,3 & 12,4 & 54,6 & 489,9 & 32527,15 & 14350,14 & 15374,14 & 536805405,29 \\
\hline $\operatorname{dim}\left(\mathcal{M}_{\text {JI,T }}\right), \quad \operatorname{dim}(\phi)$ & 7,3 & 15,7 & 54,9 & 478,9 & 32113,15 & 14133,59 & 14982,45 & 536613988,39 \\
\hline $\operatorname{dim}\left(\mathcal{M}_{\mathrm{AJI}, \mathrm{T}}\right), \operatorname{dim}(\phi)$ & 7,3 & 15,7 & 54,9 & 478,9 & 32113,15 & 14133,59 & 14982,45 & 536613988,39 \\
\hline $\operatorname{dim}\left(\mathcal{M}_{\mathrm{AJIE}, \mathrm{T}}\right), \operatorname{dim}(\phi)$ & 7,3 & 15,7 & 57,9 & 489,9 & 32527,15 & 14395,59 & 15405,45 & 536805415,39 \\
\hline $\operatorname{dim}\left(\mathcal{M}_{T}\right), \quad \operatorname{dim}(\phi)$ & 7,3 & 15,7 & 63,15 & 511,31 & 32767,255 & 16383,2047 & 16383,1023 & 536870911,65535 \\
\hline
\end{tabular}

DIMENSIONS OF MODEL CLASS AND THEIR CORRESPONDING RECEIVER (OUTPUT) DISTRIBUTION CLASS FOR SPECIFIC EXAMPLE TOPOLOGIES. THE VALUES FOR $\mathcal{M}_{\text {JI,T }}$ AND $\mathcal{M}_{\text {AJI,T }}$ (IN GREY) ARE IDENTICAL.

The number of elements of $\mathbf{p}$ specified by these $\rho$-values is given by the number of distinct events of the form $\cap_{v \in T(V)} Z_{v}=1$, i.e., the number of subtrees of $T$ that are rooted at 0 . Let $t_{T}(i)$ be the number of connected subtrees of $T$ rooted at $i$. Then $t_{T}(i)$ can be determined for a given $T$ with the following recursion relation:

$$
t_{T}(i)= \begin{cases}1 & \text { if } i \in R \\ \prod_{j \in c(i)}\left(t_{T}(j)+1\right) & \text { otherwise. }\end{cases}
$$

Of the $t_{T}(0)$ subtree events of interest here, $t_{T}(0)-1$ have probabilities specified by the $\rho$-values $\left(\operatorname{since} \operatorname{Pr}\left(X_{0}=1\right)=1\right.$ always).

Hence $t_{T}(0)-1$ of the elements of $\mathbf{p}$ fix the output, and these are determined by $N_{\rho}$ parameters. The remaining $d-\left(t_{T}(0)-1\right)$ elements of $\mathbf{p}$ are free. Thus, the set of JI models for a topology $T$ with $n$ links has dimension:

$$
\begin{aligned}
\operatorname{dim}\left(\mathcal{M}_{\mathrm{JI}, \mathrm{T}}\right) & =N_{\rho}+d-\left(t_{T}(0)-1\right) \\
& =N_{\rho}+2^{n}-t_{T}(0) .
\end{aligned}
$$

In the binary case this simplifies to:

$$
\operatorname{dim}\left(\mathcal{M}_{\mathrm{JI}, 2, \mathrm{~T}}\right)=3(n-m)+1+2^{n}-t_{T}(0) .
$$

The dimension of the class of output distributions induced by $\mathcal{M}_{\mathrm{JI}, \mathrm{T}}$ can be shown to be (see Proposition 7 in the Appendix)

$$
\operatorname{dim}\left(\phi\left(\mathcal{M}_{\mathrm{JI}, \mathrm{T}}\right)\right)=N_{\rho}-(n-m) .
$$

That is, given $\phi\left(f_{\mathbf{Z}}\right) \in \phi\left(\mathcal{M}_{\mathrm{JI}, \mathrm{T}}\right)$, we can determine $f_{\mathbf{X}}$ only up to $n-m$ degrees of freedom - this is the unidentifiability of JI models to which we previously referred.

$\mathcal{M}_{\mathrm{AJI}}$ has the same dimension as $\mathcal{M}_{\mathrm{JI}}$ models, since the subset of $\mathcal{M}_{\mathrm{AJI}}$ where every sibling set is perfectly disagreeing is the same dimension as $\mathcal{M}_{\mathrm{JI}}$ - the only constraints are that various shared transmission values are not equal.

The dimension of $\mathcal{M}_{\mathrm{AJIE}}$ can be determined similarly to $\mathcal{M}_{\mathrm{CE}}$, giving:

$$
\begin{aligned}
\operatorname{dim}\left(\mathcal{M}_{\mathrm{AJIE}, \mathrm{T}}\right) & =N_{\rho}-(n-m)+d-d_{R} \\
& =N_{\rho}-n+m+2^{n}-2^{m} .
\end{aligned}
$$

\section{Comparisons}

Table I lists concrete examples of model classes for specific topologies. The restrictive nature of $\mathcal{M}_{\mathrm{C}, \mathrm{T}}$ compared to the other classes is striking, as is the small size of the dimension gap between $\mathcal{M}_{\mathrm{AJIE}, \mathrm{T}}$ and that of the full space, $\mathcal{M}_{T}$.

The special case of JI models with binary topologies is instructive. It follows from Theorem 3 in Section VII-C that when $0<l_{i}<1$ does not hold, they provide an explicit example of topologically determinate models outside of $\mathcal{M}_{\mathrm{CE}}$ (provided no invisible sub-paths). In the binary case it follows that $\operatorname{dim}\left(\mathcal{M}_{\mathrm{CE}, \mathrm{T}} \cap \mathcal{M}_{\mathrm{JI}, \mathrm{T}}\right)=\operatorname{dim}\left(\mathcal{M}_{\mathrm{JI}}\right)$, which implies (also in the binary case) that $\operatorname{dim}\left(\mathcal{M}_{\mathrm{JI}, \mathrm{T}}\right) \leq \operatorname{dim}\left(\mathcal{M}_{\mathrm{CE}, \mathrm{T}}\right)$. Jump Independence is more restrictive than Classical Equivalence for binary models, because it places restrictions on the full joint distribution of $\mathbf{X}$, rather than just $\mathbf{X}_{R}$. This makes $\mathcal{M}_{\mathrm{JI}}$ more desirable than $\mathcal{M}_{\mathrm{CE}}$ for loss modelling - it doesn't depend on which nodes are chosen to be receivers, since the JI structure is present everywhere in the tree.

For non-binary topologies on the other hand, a new factor enters in which this time favors $\mathcal{M}_{\mathrm{JI}}$ over $\mathcal{M}_{\mathrm{CE}}$. This is because $\mathcal{M}_{\mathrm{JI}}$ allows a fully general dependency between siblings, whereas $\mathcal{M}_{\mathrm{CE}}$ places restrictions to keep the network appearing 
classical. (In the binary case, this has no dimensional effect. In the examples in the figure however, we see that overall the two are of roughly comparable dimension.) More importantly, in the general case of non-binary trees, $\mathcal{M}_{\mathrm{AJI}} \cap \mathcal{M}_{\mathrm{CE}}$ forms only a negligible subset of $\mathcal{M}_{\mathrm{AJI}}$, because a typical member of $\mathcal{M}_{\mathrm{AJI}}$ will be of 'disagreeable' type, which cannot be in $\mathcal{M}_{\mathrm{CE}}$.

\section{CONCLUSiON}

We have addressed the problem of inferring the detailed topology of a measurement tree induced by multicast probing, based on the joint distribution of loss measurements made by receivers stationed at its leaves. Previous work has shown this to be feasible under a strong simplifying assumption: that link loss processes are spatially and temporally independent. In particular, exploiting spatial independence has been the foundation of prior work in the area, not only because of its tractability, but also because within this class the topology is identifiable. The challenge here is to ask what other classes may also have this topological identifiability property.

We formalized the problem through the notion of topological determinism, which allows the topology to be identifiable, even if the full vector link loss process defining the model is not. Using this notion we were able to include spatial dependency for the first time though the $\mathcal{M}_{\mathrm{CE}}$ class, and we showed that the SLTD algorithm can recover the topology despite the spatial structure. In doing so we exploited a simple but general 'equivalence' principle (Proposition 1), which in a sense uses the lack of link process identifiability to extend the range of models for which any specific algorithm is successful. We note that the $\mathcal{M}_{\mathrm{CE}}$ is the largest class which both 'looks classical' and it still identifiable. The observables here can be modelled within the class of latent-variable tree models [31], where the LTM is simply the equivalent classical model.

Although natural, $\mathcal{M}_{\mathrm{CE}}$ is constrained by its strong ties to classical models, is not constructive, and lacks motivation in terms of real networks. We sought therefore to define a model class capable of richer dependencies through an explicit structure that was also physically meaningful in terms of network loss processes. We introduced the Jump Independent class $\mathcal{M}_{\mathrm{JI}}$ as one which offers a fully general local structure, combined with a 'no action at a distance' global structure. We believe JI models provide a realistic and sensible way to limit the dependency structure of network models. By restricting this class slightly in order to remove inherently non-identifiable features such as invisible links or subpaths, we arrived at a subclass, $\mathcal{M}_{\mathrm{AJI}}$, which is topologically determinate but has the same size (in the sense of the parameter space dimension) as $\mathcal{M}_{\mathrm{JI}}$ itself. We then defined an algorithm, SLTD2, based on the use of agreement sets which process receiver-path shared transmission probabilities in a new way, which is capable of recovering the topology. Finally, we employed the equivalence principle to prove that SLTD2 also works for models in the corresponding $\mathcal{M}_{\mathrm{AJIE}}$ class, which includes CE, and furthermore showed that $\mathcal{M}_{\mathrm{AJIE}}$ has high dimension in the space of all models. Thus SLTD2 is also an alternative algorithm for classical models.

In summary, the paper moves loss-based multicast topology inference from being restricted to a very small set of models, the classical models, to a very large class. This class is broad enough to cope with the local spatial dependencies which we know exist in real networks now, including those inherent to the use of multicast probing (Section II), or which may emerge in the future, for example through the spread of Software Defined Networking. Inference methods based on these classes will be valid even if network bottlenecks create loss which is both high and highly dependent, even negatively dependent. They will be computationally feasible because SLTD2 is still based entirely on the set of pairwise shared loss measurements only, just as in the classical case.

In future work, we intend to develop and evaluate practical methods to accurately recover from data the topology of models in $\mathcal{M}_{\mathrm{AJI}}$. There are a number of challenges here. These include the selection of a well defined metric enabling errors in topology to be meaningfully measured, the ability to randomly select topologies from the set of trees with given $m$ and various degree constraints, to select models uniformly from $\mathcal{M}_{\mathrm{AJI}}$, and the ability to estimate agreement sets. The latter is non-trivial as they are defined by equality relations which do not hold for finite data based estimates.

\section{APPENDIX}

Proof of Theorem 3. We will show that $\operatorname{Pr}\left(\bigcap_{r \in U}\left\{X_{r}=1\right\}\right)=\operatorname{Pr}\left(\bigcap_{r \in U}\left\{X_{r}^{\prime}=1\right\}\right)$ for all $U \subseteq R$. This is sufficient to show that the full joint distributions are the same because of the sufficiency of passage probabilities.

Consider an arbitrary $U \subseteq R$ and let the corresponding induced tree be $T(U)=(v(U), \ell(U))$. From Proposition 4

$$
\operatorname{Pr}\left(\bigcap_{r \in U}\left\{X_{r}=1\right\}\right)=\prod_{i \in O(T(U))} \rho_{i} \prod_{(i, j) \in J(T(U))} \rho_{i, j},
$$

and from the properties of classical models we know

$$
\operatorname{Pr}\left(\bigcap_{r \in U}\left\{X_{r}^{\prime}=1\right\}\right)=\prod_{i \in \ell(U)} l_{i}
$$

To show that the right hand sides of (25) and (24) are equal we will count the powers of $\rho_{i}$ and $\rho_{i, j}$ in the product $P=\prod_{i \in \ell(U)} l_{i}$ for each value of $i$ and pair $\{i, j\}$ respectively. Note that in the definition of $l_{i}, \rho_{i}$ only appears in $l_{s(i)}$ and $l_{f(i)}$ while $\rho_{i, j}$ appears in $l_{f(i)}, l_{i}$ and $l_{j}$. 
Powers of $\rho_{i}$ : There are five cases:

$\underline{i=1}: \quad \rho_{1}$ appears in $l_{1}$ with power +1 and appears nowhere else, so it appears with power +1 in $P$. Note $1 \in O(T(U))$. $i, s(i) \in \ell(U)$ : That is, $i \in J(T(U))$. Then $\rho_{i}$ appears in $l_{s(i)}$ with power -1 and $l_{f(i)}$ with power +1 so does not appear in the product $P$.

$i \in \ell(U), s(i) \notin \ell(U)$ : That is, $i \in O(T(U))$. Then $\rho_{i}$ appears in $l_{f(i)}$ with power +1 .

$\overline{i \notin \ell(U), s(i) \in \ell(U)}$ : That is, $s(i) \in O(T(U))$. Then $\rho_{i}$ appears in $l_{s(i)}$ with power -1 and $l_{f(i)}$ with power +1 , so $\rho_{i}$ does not appear in $P$.

$i, s(i) \notin \ell(U)$ : Then $\rho_{i}$ does not appear in the product (since $l_{s(i)}$ and $l_{f(i)}$ are not in it).

Powers of $\rho_{i, j}$ : If $(i, j) \in J(T(U))$ then $\rho_{i, j}$ appears in $l_{i}, l_{j}$ and $l_{f(i)}$ with powers of $+1,+1$ and -1 respectively. Thus $\rho_{i, j}$ appears in $P$ with power +1 . If $(i, j) \notin J(T(U))$ then $\rho_{i, j}$ does not appear in the product.

By combining the above we find that the powers of $\rho_{i}$ and $\rho_{(i, j)}$ match those of the right hand side of (25).

Proof of Lemma 4. We start by showing that a set is an AAS $\Leftrightarrow$ it is a subset of an IAAS.

$(\Leftarrow)$ Assume $D$ is an IAAS. Let $i, j \in D$ (so $i, j$ are siblings). Consider a node $k \in R, k \neq i, j$. There are 2 cases:

- $k \in d(f(i))$. Let $k^{\prime} \in(a(k) \cup k) \cap c(f(i))$. The node $k^{\prime}$ is not necessarily distinct from $k$, but must be distinct from $i$, since $i, k \in R$. Now, $S_{i, k}=S_{i, k^{\prime}}=S_{j, k^{\prime}}=S_{j, k}$ from Corollary 1 and the internally agreeing property, so $k \in A_{i, j}$.

- $k \notin d(f(i))$. Then $b_{i, k}=b_{j, k}$ and $a(i) \cap c\left(b_{i, k}\right)=a(j) \cap c\left(b_{j, k}\right)$ so $S_{i, k}=S_{j, k}$ so $k \in A_{i, j}$.

Thus $k \in A_{i, j}$ for all $k \neq i, j$, so $D$ is an AAS, as is any subset of $D$. Consequently, any subset of an IAAS is an AAS.

$(\Rightarrow)$ Let $D$ be an AAS. Assume $D$ is not a subset of an available set. Then there exists $i, j \in D$ such that $i, j$ are not siblings and without loss of generality $j \notin d(f(i))$. Then pick $k \in d(f(i))$ and $b_{i, j} \rightarrow b_{i, k}$ not being invisible implies that $S_{i, k} \neq S_{j, k}$ by Proposition 5 , so $k \notin A_{i, j}$ and thus $D$ cannot be an AAS, a contradiction.

We have shown that a set $D$ is an AAS $\Leftrightarrow D$ is a subset of an available set. It follows that $D$ is a MAAS $\Leftrightarrow D$ is an available set.

Proof of Lemma 5. $(\Rightarrow)$ Assume $D$ is an IDASS with $|D| \geq 3$. Pick arbitrary $i, j \in D$. Now consider a $k \in R \backslash D$. Since $D$ is an available sibling set, $b_{i, k}=b_{j, k}$ and $c\left(b_{i, k}\right) \cap a(i)=c\left(b_{i, k}\right) \cap a(j)$ so $S_{i, k}=S_{j, k}$ by Corollary 1 and thus $k \in A_{i, j}$. Now instead consider $k \in D$. Then $S_{i, k} \neq S_{j, k}$ since $D$ is an IDSS and thus $k \notin A_{i, j}$. So $A_{i, j}=R \backslash D$ for any $i, j \in D$, and hence $D$ is an EAS.

$(\Leftarrow)$ Let $D$ be an EAS. Thus $|D| \geq 3$. Assume $D$ is not an available sibling set. There are two cases:

1) $D$ is a proper subset of a sibling set $J$. Let $i, j \in D$ and then there must exist $k \in d(f(i)) \cap R \backslash D$. Since $D$ is externallyagreeing it cannot be all-agreeing, and so neither can $J \supset D$. Hence $S_{i, k} \neq S_{j, k}$, and so $k \notin A_{i, j}$, a contradiction with $D$ being externally-agreeing.

2) $D$ contains two nodes which aren't siblings of each other, $i, j$ say. Without loss of generality, assume $j \notin d(f(i))$. Then consider a $k \in d(f(i)) \cap R$ with $k \neq i$. Since $j \notin d(f(i)), b_{i, j}=b_{j, k}$ and $S_{i, k}=S_{j, k}$, so $j \in A_{i, k}$. Thus if $k \in D$ then $D$ is not externally-agreeing, a contradiction. However if $k \notin D$ then $S_{i, k}=S_{j, k}$ by the definition of externally-agreeing, but this implies that $b_{i, j} \rightarrow b_{j, k}$ is an invisible path, again a contradiction.

Thus, assuming $D$ is an EAS implies that it is an available sibling set.

Proposition 7. The dimension of the space of output distributions corresponding to $\mathcal{M}_{\mathrm{JI}}$ is

$$
\operatorname{dim}\left(\phi\left(\mathcal{M}_{\mathrm{JI}, \mathrm{T}}\right)\right)=N_{\rho}-n+m .
$$

Proof. For each $j \in R$ define

$$
Q_{j}=\operatorname{Pr}\left(X_{j}=1\right)=\prod_{i \in 0 \rightarrow j} \rho_{i} .
$$

For each $j \in V \backslash R$, for each $D \subset c(j)$ with $|D| \geq 2$ define

$$
P_{D}=\frac{\rho_{0 \rightarrow j} \rho_{D}}{\prod_{i \in D} \rho_{0 \rightarrow i}} .
$$

We can equivalently define $P_{D}$ as follows. Pick a set $U \subset R$ such that $|U|=|D|$ and each element of $U$ is a descendent of a different element of $D$. Then

$$
P_{D}=\frac{\operatorname{Pr}\left(\cap_{i \in U}\left\{X_{i}=1\right\}\right)}{\prod_{j \in U} Q_{j}} .
$$

This shows that we can determine $P_{D}$ from receiver observations. 
Define the vector $\mathbf{P}$ which is just the $P_{D}$ in lexicographic order with respect to each $D$ and define $\mathbf{Q}$ as the $Q_{j}$ in increasing order with respect to each $j$. Then we claim that for each $U \subset R$

$$
\operatorname{Pr}\left(\bigcap_{i \in U}\left\{X_{i}=1\right\}\right)=\prod_{i \in U} Q_{i} \prod_{i:|c(i) \cap T(U)|>1} P_{c(i) \cap T(U)} .
$$

By Proposition 4, we can prove (30) by showing

$$
\prod_{i \in T(U) \backslash U} \rho_{c(i) \cap T(U)}=\prod_{i \in U} Q_{i} \prod_{i:|c(i) \cap T(U)|>1} P_{c(i) \cap T(U)}
$$

We show this by counting the powers of $\rho_{D}$ in the product on each side of (31). First note that only $T(U)$ matters here, no other nodes or links from $T$ appear, in particular if $D \not \subset T(U)$ then it doesn't appear in either product. Consequently for convenience what follows is written as if $T(U)$ is the full tree. We break the task down into cases based on considering each $D$ separately using (27) and (28).

1) If $|D|>1$ is a sibling set then $\rho_{D}$ appears once on each side.

2) If $|D|>1$ is a not a sibling set then $\rho_{D}$ does not appear on either side.

3) If $|D|=1$, say $|D|=\{j\}$, but $D$ is not a sibling set, then $\rho_{j}$ does not appear on the left side. On the right side it appears:

- With power +1 in $\prod_{i \in U} Q_{i}$ for each $i \in R \cap(d(j) \cup j)$;

- with power -1 in $\prod_{i:|c(i)|>1} P_{c(i)}$ when $i=f(j)$; and

- with power $1-|c(i)|$ in $\prod_{i:|c(i)|>1} P_{c(i)}$ for each $i \in d(j) \cup j$ and $c(i) \cap d(j)>1$.

But

$$
\begin{aligned}
\sum_{i \in j \cup d(j): c(i)>1} 1-|c(i)| & =-\sum_{i \in j \cup d(j)}|c(i)|-1 \\
& =-(|R \cap(d(j) \cup j)|-1)
\end{aligned}
$$

because $|c(i)|-1$ is essentially the increase in the number of branches of the subtree $d(j)$ at node $i$. So in total, $\rho_{j}$ appears with power 0 on the right hand side as well.

4) This just leaves the case where $D=\{j\}$ is a sibling set. On the left hand side of (31) it appears exactly once. The right hand side is identical to when $j$ is not a sibling set, except it doesn't appear with power -1 in $\prod_{i:|c(i)|>1} P_{c(i)}$ when $i=f(j)$; so overall it appears with power +1 on the right hand side as well.

We have just shown that (30) is true, which means that $\mathbf{P}, \mathbf{Q}$ together determine the output $\mathbf{p}_{R}$. But we already know that the reverse is true, since $\mathbf{p}_{R}$ specifies the $\mathbf{Q}$ by definition, and (29) shows that $\mathbf{p}_{R}$ determines $P_{D}$ for any set of siblings $D$. Thus there is a 1-1 relationship between $(\mathbf{P}, \mathbf{Q})$ and the output distribution $f_{R} \in \phi\left(\mathcal{M}_{\mathrm{JI}, \mathrm{T}}\right)$, specified by $\mathbf{p}_{R}$. Now we will show that this is a minimal parametrization by showing that there is no way to determine any one of the parameters in $\mathbf{P}, \mathbf{Q}$ from the union of all the others.

First, assume we know $\mathbf{Q}$ and $P_{D}$ for all sibling sets $D$ other than the set $D^{\prime}$. The equations relating the known $P_{D}$ and each $Q_{i}$ to the $\rho$-values do not include $\rho_{D^{\prime}}$, so there is no way to determine the value of

$$
P_{D^{\prime}}=\frac{\rho_{0 \rightarrow j} \rho_{D^{\prime}}}{\prod_{i \in D^{\prime}} \rho_{0 \rightarrow i}}
$$

since each $\rho$-value can be set independently.

Next, assume we know $\mathbf{P}$ and all-but-one element of $\mathbf{Q}, Q_{k}$ say. Consider the set of relations between the vector of $\rho$-values and $\mathbf{P}, \mathbf{Q}$. The relations involving $\rho_{k}, k \in R$, are: the relation giving $Q_{k}$ and the relations giving $P_{D}$ for each $D \supset k$. If $\rho_{k}$ is multiplied by a factor $r$ and each $\rho_{D}$ with $D \supset k$ is multiplied by that same factor $r$ then $\mathbf{P}$ and the $Q$ s other than $Q_{k}$ will stay the same, but $Q_{k}$ will be $r$ times larger. Consequently, in the general case there is no way to determine $Q_{k}$ from $\mathbf{P}$ and the other $Q_{j}, j \neq k$.

Thus, the set of parameters $\mathbf{P}, \mathbf{Q}$ is $1-1$ with output distributions, and it has no redundancy. There are $N_{\rho}-n$ parameters in $\mathbf{P}$ (because the number of possible sibling sets with $|D|=1$ over the tree is just the number of links, and these are excluded from $\mathbf{P}$ ) and $|R|=m$ parameters in $\mathbf{Q}$, so the dimension of $\phi\left(\mathcal{M}_{\mathrm{JI}}\right)$ is $N_{\rho}-n+m$.

Note that the difference in the number of degrees of freedom in setting the $\rho$-values, and the dimension of the output, is just $n-m$, the number of interior nodes. That is, the space of vectors of $\rho$-values corresponding to any given output has dimension $n-m$. This is a measure of the part of the non-identifiability at the model level associated with the $\rho$ values, and can be viewed as one unknown per internal-node.

\section{ACKNOWLEDGEMENT}

This work was supported by Australian Research Council's Discovery Projects funding scheme (\#DP120102834). The authors are grateful to François Baccelli for suggesting this research problem and for highlighting its importance for inference in access networks. 


\section{REFERENCES}

[1] R. Caceres, N. Duffield, J. Horowitz, D. Towsley, and T. Bu, "Multicast-Based Inference of Network Internal Loss Characteristics: Accuracy of Packet Estimation," in IEEE INFOCOM'99, New York, NY., Mar. 1999.

[2] R. Caceres, N. Duffield, J. Horowitz, and D. Towsley, "Multicast-Based Inference of Network-Internal Loss Characteristics," IEEE Transactions on Information Theory, vol. 45, pp. 2462-2480, 1999.

[3] A. Adams, T. Bu, T. Caceres, N. Duffield, T. Friedman, J. Horowitz, F. L. Presti, S. Moon, V. Paxson, and D. Towsley, "The use of End-to-end Multicast Measurements for Characterising Internal Network Behavior," IEEE Communications Magazine, special issue on "Network Traffic Measurements and Experiments", vol. 38, no. 5, pp. 152-158, May 2000.

[4] N. Duffield and F. L. Presti, "Multicast Inference of Packet Delay Variance at Interior Network Links," in IEEE INFOCOM, Tel Aviv, Israel, Mar. 2000, pp. 1351-1360.

[5] N. Duffield, J. Horowitz, D. Towsley, W. Wei, and T. Friedman, "Multicast-based loss inference with missing data," IEEE Journal on Selected Areas of Communications, vol. 20, no. 4, pp. 700-713, 2002.

[6] F. L. Presti, N. G. Duffield, J. Horowitz, and D. Towsley, "Multicast-based inference of network-internal delay distributions," IEEE/ACM Transactions on Networking, vol. 10, no. 6, pp. 761-775, 2002

[7] N. Duffield, J. Horowitz, F. L. Presti, and D. Towsley, "Multicast Topology Inference from Measured End-to-End Loss," IEEE Transactions in Information Theory, vol. 48, no. 1, pp. 26-45, 2002.

[8] G. Liang and B. Yu, "Maximum Pseudo Likelihood Estimation in Network Tomography," IEEE Trans. on Signal Processing (Special Issue on Data Networks), vol. 51, no. 8, pp. 2043-2053, 2003.

[9] N. Duffield, J. Horowitz, F. L. Presti, and D. Towsley, "Explicit loss inference in multicast tomography," IEEE Transactions in Information Theory, vol. 52, no. 8, pp. 3852-3855, 2006.

[10] S. Ratnasamy and S. McCanne, "Inference of Multicast Routing Trees and Bottleneck Bandwidths Using End-to-end Measurements," in IEEE INFOCOM'99, 1999, pp. 353-360. [Online]. Available: citeseer.ist.psu.edu/ratnasamy99inference.html

[11] M. Coates and R. Nowak, "Network Loss Inference using Unicast End-to-End Measurement," in ITC Conference on IP Traffic, Modelling and Management, Monterey, CA, Sep. 2000.

[12] R. Caceres, N. Duffield, J. Horowitz, F. L. Presti, and D. Towsley, "Loss-Based Inference of Multicast Network Topology," in IEEE Conference on Decision and Control, Pheonix, Arizona, Dec. 1999, pp. 371-379. [Online]. Available: citeseer.ist.psu.edu/caceres98multicastbased.html

[13] M. Coates, R. Castro, M. Gadhiok, R. King, Y. Tsang, and R. Nowak, "Maximum Likelihood Network Topology Identification from Edge-based Unicast Measurements," in Proc ACM Sigmetrics, Marina Del Rey, CA, June 2002, pp. 11-20.

[14] D. Ghita, K. Argyraki, and P. Thiran, "Network tomography on correlated links," in Proceedings of the 10th annual conference on Internet measurement. ACM, 2010, pp. 225-238.

[15] F. Pin, D. Veitch, and B. Kauffmann, "Statistical Estimation of Delays in a Multicast Tree using Accelerated EM," Queueing Systems, vol. 66, no. 4, pp. 369-412, 2010. [Online]. Available: http://www.cubinlab.ee.unimelb.edu.au/ darryl/Publications/loss_train_CommLetters_camera.pdf

[16] N. Duffield, F. L. Presti, V. Paxson, and D. Towsley, "Inferring Link Loss Using Striped Unicast Probes," in IEEE INFOCOM, Anchorage, Alaska, April 22-26 2001, pp. 915-923. [Online]. Available: citeseer.ist.psu.edu/duffield01inferring.html

[17] Y. Tsang, M. Coates, and R. Nowak, "Network Delay Tomography," IEEE Trans. on Signal Processing (Special Issue on Data Networks), vol. 51, no. 8, pp. 2125-2136, 2003.

[18] M.-F. Shih and A. O. Hero, "Unicast-Based Inference of Network Link Delay Distributions With Finite Mixture Models," IEEE Trans. on Signal Processing (Special Issue on Data Networks), vol. 51, no. 8, pp. 2219-2228, 2003.

[19] E. Lawrence, G. Michailidis, and V. N. Nair, "Statistical inverse problems in active network tomography," in Complex Datasets and Inverse Problems: Tomography, Networks and Beyond, IMS Lecture Notes-Monograph Series. IMS, 2007, vol. 54, pp. 24-44.

[20] D. Ghita, H. Nguyen, M. Kurant, K. Argyraki, and P. Thiran, "Netscope: practical network loss tomography," in INFOCOM, 2010 Proceedings IEEE. IEEE, 2010, pp. 1-9.

[21] N. Duffield, "Network tomography of binary network performance characteristics," IEEE Transactions on Information Theory, vol. 52, no. 12, pp. 5373-5388, December 2006.

[22] H. Nguyen and P. Thiran, "The Boolean Solution to the Congested IP Link Location Problem," in INFOCOM, 2007 Proceedings IEEE. IEEE, 2007.

[23] D. Ghita, C. Karakus, K. Argyraki, and P. Thiran, "Shifting network tomography toward a practical goal," in Proceedings of the Seventh COnference on emerging Networking EXperiments and Technologies. ACM, 2011, p. 24.

[24] D. Ghita, K. Argyraki, and P. Thiran, "Rethinking boolean network tomography," Technical report, EPFL, Tech. Rep., 2011.

[25] H. Nguyen and R. Zheng, "A binary independent component analysis approach to tree topology inference," Signal Processing, IEEE Transactions on, vol. 61, no. 12, pp. 3071-3080, 2013.

[26] P. Sattari, A. Markopoulou, and C. Fragouli, "Multiple source multiple destination topology inference using network coding," in Network Coding, Theory, and Applications, 2009. NetCod'09. Workshop on. IEEE, 2009, pp. 36-41.

[27] R. Sherwood, A. Bender, and N. Spring, "Discarte: a disjunctive internet cartographer," in ACM SIGCOMM Computer Communication Review, vol. 38, no. 4. ACM, 2008, pp. 303-314.

[28] J. Ni, H. Xie, S. Tatikonda, and Y. R. Yang, "Efficient and dynamic routing topology inference from end-to-end measurements," IEEE/ACM Transactions on Networking (TON), vol. 18, no. 1, pp. 123-135, 2010.

[29] B. Eriksson, G. Dasarathy, P. Barford, and R. Nowak, "Efficient network tomography for internet topology discovery," IEEE/ACM Transactions on Networking (TON), vol. 20, no. 3, pp. 931-943, 2012.

[30] D. Geiger, T. Verma, J. Pearl et al., "Identifying independence in bayesian networks," Networks, vol. 20, no. 5, pp. 507-534, 1990.

[31] R. Mourad, C. Sinoquet, N. L. Zhang, T. Liu, P. Leray et al., "A survey on latent tree models and applications.” J. Artif. Intell. Res.(JAIR), vol. 47, pp. 157-203, 2013.

[32] Y. Wang, N. L. Zhang, and T. Chen, "Latent tree models and approximate inference in bayesian networks," Journal of Artificial Intelligence Research, pp. 879-900, 2008.

[33] D. Connolly, "Constructing hidden variables in baysesian networks via conceptual clustering," in Proceedings of the Tenth International Conference on Machine Learning, 2014, pp. 65-72. 